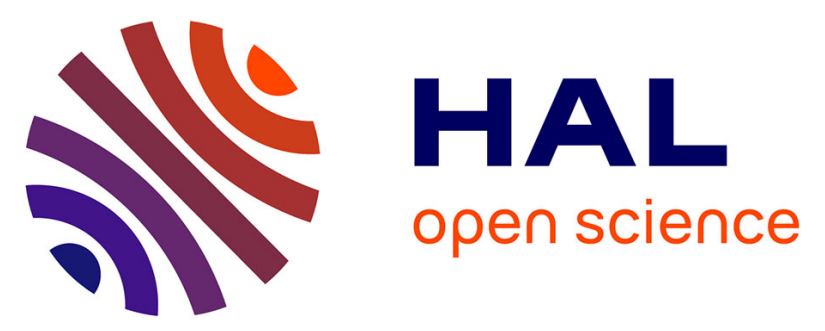

\title{
Robustness analysis and convergence of empirical finite-time ruin probabilities and estimation risk solvency margin.
}

\author{
Stéphane Loisel, Christian Mazza, Didier Rullière
}

\section{To cite this version:}

Stéphane Loisel, Christian Mazza, Didier Rullière. Robustness analysis and convergence of empirical finite-time ruin probabilities and estimation risk solvency margin.. Insurance: Mathematics and Economics, 2008, 42 (2), pp.746-762. 10.1016/j.insmatheco.2007.08.007 . hal-00168714

\section{HAL Id: hal-00168714 https://hal.science/hal-00168714}

Submitted on 29 Aug 2007

HAL is a multi-disciplinary open access archive for the deposit and dissemination of scientific research documents, whether they are published or not. The documents may come from teaching and research institutions in France or abroad, or from public or private research centers.
L'archive ouverte pluridisciplinaire HAL, est destinée au dépôt et à la diffusion de documents scientifiques de niveau recherche, publiés ou non, émanant des établissements d'enseignement et de recherche français ou étrangers, des laboratoires publics ou privés. 


\title{
Institut de Science Financière et d'Assurances
}

\section{Les Cahiers de Recherche de I'ISFA}

\author{
ROBUSTNESS ANALYSIS AND CONVERGENCE OF EMPIRICAL FINITE-TIME RUIN \\ PROBABILITIES AND ESTIMATION RISK SOLVENCY MARGIN
}

Stéphane Loisel

Christian Mazza

Didier Rullière

Cahier de Recherche WP 2033 (2006)

Université Claude Bernard Lyon 1 


\title{
Robustness analysis and convergence of empirical finite-time ruin probabilities and estimation risk solvency margin
}

\author{
Stéphane Loisel, \\ Université Claude Bernard Lyon 1 - Ecole ISFA - 50, avenue Tony Garnier - \\ F-69366 Lyon Cedex 07 \\ Christian Mazza, \\ Department of Mathematics, University of Fribourg, Pérolles, Chemin du Musée \\ 23, CH-1700 Fribourg, Switzerland \\ Didier Rullière*. \\ Université Claude Bernard Lyon 1 - Ecole ISFA - 50, avenue Tony Garnier - \\ F-69366 Lyon Cedex 07
}

\begin{abstract}
We consider the classical risk model and carry out a sensitivity and robustness analysis of finite-time ruin probabilities. We provide algorithms to compute the related influence functions. We also prove the weak convergence of a sequence of empirical finite-time ruin probabilities starting from zero initial reserve toward a Gaussian random variable. We define the concepts of reliable finite-time ruin probability as a Value-at-Risk of the estimator of the finite-time ruin probability. To control this robust risk measure, an additional initial reserve is needed and called Estimation Risk Solvency Margin (ERSM). We apply our results to show how portfolio experience could be rewarded by cut-offs in solvency capital requirements. An application to catastrophe contamination and numerical examples are also developed.
\end{abstract}

Key words: Finite-time ruin probability, robustness, Solvency II, reliable ruin probability, asymptotic Normality, influence function, Estimation Risk Solvency Margin (ERSM).

JEL Classification codes: G22, C60.

* Corresponding author. Tel. +3343728 74 38, fax +3343728 7632

Email addresses: Stephane.Loisel@univ-lyon1.fr (Stéphane Loisel), christian.mazza@unifr.ch (Christian Mazza), Didier.Rulliere@univ-lyon1.fr (Didier Rullière).

Cahiers de Recherche de l'ISFA, WP 2033 (2006) 


\section{Introduction}

No matter whether it is for risk capital allocation, for solvency requirements, or just for risk measurement, most actuaries traditionally start by fitting the corresponding data with some distribution using log-likelihood maximization, moment-based methods, or other statistical procedures, and then compute the probability of ruin, the Value-at-Risk, or some relevant risk-related quantity based on probabilistic models involving the fitted distribution. Robust statistics is a huge field, extensively studied in the seventies and in the eighties, in particular by Hampel (1974) and Huber (1981), and provides powerful concepts for sensitivity studies. Recently, Marceau and Rioux (2001) pointed out the importance of robust statistical methods in risk theory, and provided sensitivity results for infinite-time ruin probabilities. Actuaries are nowadays more interested in finite-time ruin probabilities, within a time-horizon between 1 and 10 years. Robust estimation of finitetime ruin probabilities is really in the spirit of pillar I of Solvency II. Robust CVaRs were used in different papers for portfolio selection for example. Finite-time ruin probabilities were studied in several papers, in particular Picard and Lefèvre (1997), Rullière and Loisel (2004), De Vylder (1999), and Ignatov, Kaishev and Krachunov (2001). But surprisingly, no robustness analysis of the finite-time ruin probability has appeared in the literature yet to our knowledge. Similarly, asymptotic Normality of estimators of infinite-time ruin probabilities has been studied by Croux and Veraverbeke (1990) and more recently by Bening and Korolev (2000). Consistency of bootstrap estimators of finite and infinite-time ruin probabilities had also been studied by Frees (1986) and Hipp (1989). Estimation risk has been designated as one of the risks that should be taken into account in the Solvency II project. Despite this motivation, as far as we know, asymptotic Normality of estimators of finite-time ruin probabilities had neither been proved nor used to take estimation risk into account.

In this paper, we first continue on the track of Marceau and Rioux (2001) and we tackle the robustness analysis of finite-time ruin probabilities in the classical risk model. We then prove the convergence of the rescaled error on the finite-time ruin probability toward a Gaussian random variable if computations are carried out with the empirical claim amount distribution. We compute explicitly the variance of this distribution and can thus define and quantify the reliable finite-time ruin probability. This Value-at-Risk of the estimator of the finite-time ruin probability has to be controlled to cover estimation risk, which requires an additional solvency capital compare to the case where one only controls the empirical finite-time ruin probability : we define this capital as the Estimation Risk Solvency Margin (ERSM).

Our paper is organized as follows: in section 2, we recall the classical risk model and the literature about computation of "classical" finite-time ruin probabilities. We derive some sensitivity results that are going to be useful in the sequel. We also introduce the concept of influence function and briefly recall its main properties. In section 3 we compute influence functions of finite-time ruin probabilities and of some related quantities, using some formulas of Picard and Lefèvre (1997) and Rullière and Loisel (2004) as starting points. In section 4 we study some properties of the influence function, in particular large claim contamination in catastrophe risk. We use a result from Hoeffding (1948) to show the weak convergence of a sequence of empirical ruin probabilities to a Gaussian process in section 5 . In section 6 , we explain how to use the influence function and our results to 
get a more robust determination of solvency capital requirements with reliable finite-time ruin probabilities : the required capital is the sum of the capital that is required to have a probability of ruin based on empirical claim size distribution less than $\epsilon$ and of the Estimation Risk Solvency Margin (ERSM). The goal of ERSM is to take estimation risk into account in the spirit of Solvency II. The value of this margin may be easily obtained thanks to the Gaussian approximation derived in section 5. The impact of excluding some types of catastrophe risks in insurance or reinsurance treaties is also obtained with a very simple formula. Numerical examples illustrate the developed methods in section 7 . In particular, we show that the better the experience of the company about claim sizes is, the lower the estimation risk solvency margin (ERSM) is. The experience of the company about claim sizes is quantified by the number of observed claim amounts in the database. The higher this number, the smaller the ERSM.

\section{The classical risk model : sensitivity analysis and influence function}

We will consider a classical risk process $\left(R_{t}\right)_{t \geq 0}$ defined as follows : for $t \geq 0$,

$$
R_{t}=u+c t-S_{t},
$$

where $u$ is the non-negative amount of initial reserves, $c>0$ is the premium income rate. The cumulated claim amount up to time $t$ is described by the compound Poisson process

$$
S_{t}=\sum_{i=1}^{N_{t}} W_{i},
$$

where amounts of claims $W_{i}, i=1,2, \ldots$ are non-negative independent, identically-distributed random variables, distributed as $W$. As usual $S_{t}=0$ if $N_{t}=0$. The number of claims $N_{t}$ until $t \geq 0$ is modeled by an homogeneous Poisson process $\left(N_{t}\right)_{t \geq 0}$ of intensity $\lambda$. Claim amounts and arrival times are assumed to be independent.

We are interested in the robust estimation of finite-time ruin probabilities. Let us denote by $\psi(u, t)$ the probability of ruin before time $t$ with initial reserve $u$ :

$$
\psi(u, t)=\mathrm{P}\left[\exists s \in[0, t], \quad R_{s}<0 \mid R_{0}=u\right], \quad u \geq 0, t>0,
$$

and let

$$
\varphi(u, t)=1-\psi(u, t)
$$

be the probability of non-ruin within time $t$ with initial reserve $u$. As we consider finitetime ruin probabilities, no profit condition has to be satisfied from a theoretical point of view.

\subsection{Sensitivity analysis}

We show here that derivatives of the finite-time non ruin probability $\varphi(u, t)$ with respect to $c, \lambda$ or $u$ may be easily obtained as functions of derivatives of the density $f_{S_{t}}(x)$ of $S_{t}$ 
with respect to $x$ or $\lambda$. Some details or parts of proof are given in Appendix.

\subsubsection{Continuous claim amount distribution}

Consider first the case of continuous claim amount distributions. Note that derivatives of the density of the cumulated claim amount $S_{t}$ up to time $t$ can easily be obtained by differentiation of the continuous version of Panjer's formula :

$$
f_{S_{t}}(x)=\lambda \int_{0}^{x} \frac{y}{x} f_{W}(y) f_{S_{t}}(x-y) d y, \quad x>0,
$$

where $f_{S_{t}}$ and $f_{W}$ respectively are the p.d.f. of $S_{t}$ and $W$.

Proposition 1 Let $k \in \mathbb{N}$. Then for $u, c, t>0$ such that $f_{W}$ is $k$-times continuously differentiable on $[0, u+c t]$,

$$
\frac{\partial^{k}}{\partial u^{k}} \varphi(u, t)=\frac{\partial^{k}}{\partial u^{k}} f_{S_{t}}(u+c t)-\int_{0}^{t} \frac{\partial^{k}}{\partial u^{k}} f_{S_{x}}(u+c x) \varphi(0, t-x) d x .
$$

For $u, c, t>0$ such that $f_{W}$ is continuously differentiable on $[0, u+c t]$,

$$
\frac{\partial}{\partial c} \varphi(u, t)=t f_{S_{t}}(u+c t)-\int_{0}^{t} x f_{S_{t}}^{\prime}(u+c x) \varphi(0, t-x)+f_{S_{t}}(u+c x) \frac{\partial}{\partial c} \varphi(0, t-x) d x
$$

This provides a self-iterative process to determine $\varphi(0, x)$ (for $u=0)$.

In the discrete case, expressing partial derivatives of finite-time ruin probabilities in terms of derivatives of some $f_{S_{t}}$ provides natural recursive computation schemes.

\subsubsection{Discrete claim amount distribution}

In the case of integer-valued claim amounts, we can either use finite-difference calculus instead of differentiation, or study the particular behavior of $\varphi(u, t)$ as $u$ varies for example. As explained in Rullière and Loisel (2004), ruin and ruin at inventory are exactly the same, provided that the set of inventory dates $\Omega$ is chosen as

$$
\Omega=\{\tau \in] 0, t], \quad u+c \tau \in \mathbb{N} \backslash\{0\}\} .
$$

This set of inventory dates $\Omega$ depends on $u, t$ and $c$ but not on $\lambda$.

Set $x_{+}=\max (x, 0)$.

Proposition 2 The partial derivatives of order $k$ w.r.t. $\lambda$ of finite-time non-ruin probabilities starting from zero can be written as follows: 


$$
\begin{aligned}
\frac{\partial}{\partial \lambda} \varphi\left(0, \frac{n}{c}\right) & =\frac{n}{c} \mathrm{E}\left[\left(1-\frac{W+S_{n / c}}{n}\right)_{+}\right]-\frac{n}{c} \varphi\left(0, \frac{n}{c}\right), \quad k \geq 1, \\
\frac{\partial^{k}}{\partial \lambda^{k}} \varphi\left(0, \frac{n}{c}\right) & =\left(\frac{n}{c}\right)^{k} \sum_{i=0}^{k} C_{k}^{i}(-1)^{k-i} \mathrm{E}\left[\left(1-\frac{W^{* i}+S_{n / c}}{n}\right)_{+}\right], \quad k \geq 0 .
\end{aligned}
$$

Proof: $\varphi(0, t)=\mathrm{E}\left[\left(1-\frac{S_{t}}{c t}\right)_{+}\right]$, and (A.3) give the result.

Proposition 3 For $k \geq 1$, partial derivatives of order $k$ w.r.t. $\lambda$ of finite-time non-ruin probabilities can be written as follows:

$$
\frac{\partial^{k}}{\partial \lambda^{k}} \varphi(u, t)=\frac{\partial^{k}}{\partial \lambda^{k}} \mathrm{P}\left[R_{t} \geq 0\right]-\sum_{s \in \Omega} \sum_{i=0}^{k} C_{k}^{i} \frac{\partial^{i}}{\partial \lambda^{i}} \mathrm{P}\left[R_{s}=0\right] \frac{\partial^{k-i}}{\partial \lambda^{k-i}} \varphi(0, t-s) .
$$

Proof: This follows from Proposition 2 in Appendix and results of Rullière and Loisel (2004).

Remark 1 Some results concerning derivatives of ruin probabilities involve the distribution of $W+S_{t}$. This will also be the case for some results about influence functions in the next sections, in particular for propositions 15, and 16 in the case of large claim contamination. If we add a claim at time zero, we can link the involved ruin probability with a ruin probability in the so-called dual risk model, in which the risk process decreases at a deterministic rate and has upward jumps. We can also link the involved ruin probability with the probability that a classical process reaches an upper barrier. For more details, see Mazza and Rullière (2004).

\subsection{Influence functions}

It is unlikely that the "real" claim amount process is exactly the one which has been chosen for statistical inference. At best, it might correspond to a model that is close to the starting model, for example a small contamination of it. Therefore, one needs estimators that are efficient and that do not change much if a small change occurs in the inputs of the model. Estimators of this kind are called robust. The influence function, which was introduced by Hampel (1974) to study the infinitesimal behavior of real-valued functionals, is one of the main tools in robustness theory to measure the impact of a small perturbation of the model on the outputs.

Definition 1 (Influence Function (IF)) Assume that $\mathbf{T}$ is a functional of a distribution $F$. The influence function at point $x \in \mathbb{R}$ is defined as the limit (when it exists)

$$
\operatorname{IF}_{\mathrm{x}}[\mathbf{T}]=\lim _{s \downarrow 0} \frac{\mathbf{T}\left(F^{(s, x)}\right)-\mathbf{T}(F)}{s}
$$


where $F^{(s, x)}$ is defined for $x \in \mathbb{R}$ and $0<s<1$ by

$$
\text { for } u \in \mathbb{R}, \quad F^{(s, x)}(u)=s \mathbf{1}_{x \leq u}+(1-s) F(u) .
$$

In the sequel, for each quantity related to the contaminated distribution $F^{(s, x)}$, we use the exponent ${ }^{(s, x)}$. Given a random sample $X_{1}, \cdots, X_{n}$ distributed according to some distribution function $F$, let $F_{n}$ denote the associated empirical distribution. The influence function has two main uses: it allows the study of the influence of perturbations of the data on the values taken by the functional $\mathrm{T}$, and it permits, under some regularity assumptions to catch the asymptotic variance when the rescaled process weakly converges toward a Gaussian random variable

$$
\operatorname{Var}\left(\sqrt{n}\left|\mathbf{T}\left(F_{n}\right)-\mathbf{T}(F)\right|\right) \longrightarrow A(F, \mathbf{T}),
$$

as $n \rightarrow \infty$, where

$$
A(F, \mathbf{T})=\int_{\mathbb{R}}\left(\mathrm{IF}_{\mathrm{x}}[\mathbf{T}(F)]\right)^{2} F(d x)
$$

(see Huber (1981), Hampel (1974) or Hampel et al. (1986)).

\section{Computation of the influence function}

We assume here that $W$ is integer-valued, with $\mathrm{P}[W=0]=0$ (which is not restrictive, see for example De Vylder (1999) or Rullière and Loisel (2004)). In this section, we provide algorithms to compute influence functions of finite-time non-ruin probabilities and of some related quantities. Set $\Pi_{i}=\mathrm{P}[W=i], i \in \mathbb{N}$. We assume that the distribution $F$ of a single claim amount is contaminated, in the sense that we add some probability mass at point $x \in \mathbb{N} . x$ can in general be any real number, but we present here the simpler case where $x \in \mathbb{N}$ for the sake of clarity. This is also consistent with the fact that claim amounts are integer-valued in reality. As for $u, t>0$ and $x \in \mathbb{N}$,

$$
\operatorname{IF}_{\mathrm{x}}[\psi(u, t)]=-\mathrm{IF}_{\mathrm{x}}[\varphi(u, t)],
$$

we can treat symmetrically the probability of ruin or of non-ruin before $t$.

Given $j \in \mathbb{N}$ and $\tau \in \mathbb{R}$, consider the functions

$$
h_{0}(\tau)=e^{-\frac{\lambda \tau}{c}} \text { and } h_{j}(\tau)=\frac{\lambda \tau}{c j} \sum_{i=1}^{j} i \cdot \operatorname{Pr}[W=i] \cdot h_{j-i}(\tau)
$$

For $\tau>0$, we have $h_{j}(\tau)=\mathrm{P}\left[S_{\tau}=j\right]$ for $j \in \mathbb{N}$. Set $\Pi_{i}=\mathrm{P}[W=i]$ for $i \in \mathbb{N}$, with $\Pi_{0}=0$. Similarly, for $j \in \mathbb{N}$ and $\tau \in \mathbb{R} \backslash\{0\}$, one sets

$$
H_{j}(\tau)=\sum_{i=0}^{j} h_{i}(\tau) \text { and } \widetilde{H}_{j}(\tau)=\sum_{i=0}^{j} h_{i}(\tau)\left(1-\frac{i}{\tau}\right) .
$$

Then

$$
H_{j}(0)=h_{j}(0) \text { and } \widetilde{H}_{j}(0)=1-\frac{\lambda}{c} \mathrm{E}\left[W \mathbf{1}_{W \leq j}\right]
$$




$$
h_{j}(\tau)=\operatorname{Pr}\left[S_{\tau / c}=j\right], \quad H_{j}(\tau)=\operatorname{Pr}\left[S_{\tau / c} \leq j\right] \text { and } \widetilde{H}_{j}(\tau)=\frac{1}{\tau} \mathrm{E}\left[\left(\tau-S_{\frac{\tau}{c}}\right) \mathbf{1}_{S_{\frac{\tau}{c}} \leq j}\right] .
$$

\section{Proposition 4 (IF for single claim probabilities)}

$$
\mathrm{IF}_{\mathrm{x}}\left[\Pi_{i}\right]=\mathbf{1}_{x=i}-\Pi_{i}, \quad i \in \mathbb{N} .
$$

Proof: We see that $\Pi^{(s, x)}=s \mathbf{1}_{x=i}+(1-s) \Pi_{i}$, and the result is straightforward.

In order to determine the influence function of the probability of ruin, we need to give the influence function for quantities $\mathrm{P}\left[S_{t}=j\right], j \in \mathbb{N}, t \in \mathbb{R}$. Notice that for $\tau<0$ computations are formal and do not have any probabilistic meaning. Nevertheless, these formal computations will be useful for the final results, as in Rullière and Loisel (2004).

The influence function of these probabilities will be written as follows:

$$
\mathrm{IF}_{\mathrm{x}, j}(\tau)=\mathrm{IF}_{\mathrm{x}}\left[\mathrm{P}\left[S_{\tau / c}=j\right]\right] \quad j \in \mathbb{N}, \tau \in \mathbb{R}^{+} .
$$

\section{Proposition 5 (IF for aggregated claim amount probabilities)}

$$
\mathrm{IF}_{\mathrm{x}, y}(\tau)=\frac{\lambda \tau}{c} \frac{x}{y} \mathbf{1}_{x \leq y} \mathrm{P}\left[S_{\frac{\tau}{c}}=y-x\right]-\mathrm{P}\left[S_{\frac{\tau}{c}}=y\right]+\sum_{i=1}^{y} \frac{\lambda \tau}{c} \frac{i}{y} \prod_{i} \mathrm{IF}_{\mathrm{x}, y-i}(\tau) .
$$

Proof: Using Panjer's recursion, one obtains $\mathrm{P}\left[S_{\frac{\tau}{c}}=y\right]=\sum_{i=1}^{y} \frac{\lambda \tau}{c} \frac{i}{y} \Pi_{i} \mathrm{P}\left[S_{\frac{\tau}{c}}=y-i\right]$. Thus, we can obtain both $\mathrm{P}\left[S_{\frac{\tau}{c}}(s, x)=y\right]$ and $\mathrm{P}\left[S_{\frac{\tau}{c}}=y\right]$ recursively as $y$ varies. We can then either consider the difference between $\mathrm{P}\left[S_{\frac{\tau}{c}}(s, x)=y\right]$ and $\mathrm{P}\left[S_{\frac{\tau}{c}}=y\right]$ and calculate the limit when $s$ tends to zero, or directly differentiate $\mathrm{P}\left[S_{\frac{\tau}{c}}(s, x)=y\right]$ with respect to $s$, and then take $s=0$. According to Panjer's formula, the second term on the right-hand side of the equality is reduced to $\mathrm{P}\left[S_{\frac{\tau}{c}}=y\right]$.

As for $j \in \mathbb{N}$ we can define $h_{j}(\tau)$ for $\tau \in \mathbb{R}$ (even if it loses its probabilistic interpretation, see Rullière and Loisel (2004) for example), the definition of $\operatorname{IF}_{\mathrm{x}, j}(\tau)$ may be extended to the general case $\tau \in \mathbb{R}$ for $j, x \in \mathbb{N}$ simply as follows :

$$
\mathrm{IF}_{\mathrm{x}, j}(\tau)=\left.\frac{\partial}{\partial s} h_{j}^{(s, x)}(\tau)\right|_{s=0} .
$$

Proposition 6 (Algorithm for IF related to aggregate claim amounts) The following iterative scheme provides both aggregated claim amount distributions and the corresponding influence functions, for $\tau \in \mathbb{R}$ and $x, y \in \mathbb{N}$ :

$$
\begin{gathered}
\mathrm{IF}_{\mathrm{x}, y}(\tau)=\frac{\lambda \tau}{c} \frac{x}{y} \mathbf{1}_{x \leq y} h_{y-x}(\tau)-h_{y}(\tau)+\sum_{i=1}^{y} \frac{\lambda \tau}{c} \frac{i}{y} \prod_{i} \mathrm{IF}_{\mathrm{x}, y-i}(\tau), \\
h_{y}(\tau)=\sum_{i=1}^{y} \frac{\lambda \tau}{c} \frac{i}{y} \prod_{i} h_{y-i}(\tau)
\end{gathered}
$$


where $h_{0}(\tau)=e^{-\frac{\lambda \tau}{c}}$ and

$$
\operatorname{IF}_{\mathrm{x}, 0}(\tau)=\frac{\lambda \tau}{c} e^{-\frac{\lambda \tau}{c}} \mathbf{1}_{x=0}
$$

Proof: The first equation is given by Proposition 6, the second is an expression of Panjer's formula. For initial values, one can check that for $x \neq 0, \mathrm{P}\left[S_{t}=0\right]=\mathrm{P}\left[S_{t}^{(s, x)}=0\right]=$ $\mathrm{P}\left[N_{t}=0\right]$, so that $\mathrm{IF}_{\mathrm{x}, 0}(t)=0, t \in \mathbb{R}$. For $x=0, \mathrm{P}\left[S_{t}^{(s, x)}=0\right]=e^{-\lambda t(1-s)}$.

Takács's result (see Takács (1962a) and Seal (1969)) implies that

$$
\varphi\left(0, \frac{n}{c}\right)=\sum_{j=0}^{n} \frac{n-j}{n} \mathrm{P}\left[S_{\frac{n}{c}}=j\right]=\sum_{j=0}^{n} \frac{n-j}{n} h_{j}(n), \quad n \in \mathbb{N} .
$$

As a direct consequence, we get the influence function of the finite-time non-ruin probability starting from zero.

\section{Proposition 7 (IF for $\varphi(0, t)$ )}

$$
\operatorname{IF}_{\mathrm{x}}\left[\varphi\left(0, \frac{n}{c}\right)\right]=\sum_{j=0}^{n} \frac{n-j}{n} \mathrm{IF}_{\mathrm{x}, j}(n) .
$$

Proof: Differentiate $\varphi\left(0, \frac{n}{c}\right)$ for the contaminated single amount distribution, take $s=0$ and apply then equation (6).

Computations for a time $n \notin \mathbb{N}$ and an initial reserve $u \notin \mathbb{N}$ can be done by application of formulas (2.10) and (2.11) in Rullière and Loisel (2004).For the sake of clarity, we consider $u, n \in \mathbb{N}$ in the sequel. Several ways have been proposed to compute finite time ruin probabilities with initial reserves $u \in \mathbb{N}$ (see Rullière and Loisel (2004), Picard and Lefèvre (1997)).

By conditioning by the last time the process $\left(R_{s}\right)_{s \geq 0}$ reaches zero before time $t$, we get:

$$
\varphi\left(u, \frac{n}{c}\right)=H_{u+n}(n)-\sum_{k=1}^{n} h_{u+k}(k) \widetilde{H}_{n-k}(n-k) .
$$

As a direct consequence, we get the following recursive scheme for the influence function of the finite-time non-ruin probabilities $\varphi(u, t), u \in \mathbb{N}$.

Set $\operatorname{IFH}_{\mathrm{x}, j}(\tau)=\operatorname{IF}_{\mathrm{x}}\left[\tilde{H}_{j}(\tau)\right]$. Then

$$
\operatorname{IFH}_{\mathrm{x}, j}(\tau)=\sum_{i=0}^{j} \operatorname{IF}_{\mathrm{x}, i}(\tau)\left(1-\frac{i}{\tau}\right)
$$

with $\operatorname{IFH}_{\mathrm{x}, 0}(0)=0$. In particular, $\operatorname{IFH}_{\mathrm{x}, n}(n)=\operatorname{IF}_{\mathrm{x}}\left[\varphi\left(0, \frac{n}{c}\right)\right], n \in \mathbb{N}$. 
Proposition 8 (IF for $\varphi(u, t)$ - first method)

$$
\begin{aligned}
\operatorname{IF}_{\mathrm{x}}\left[\varphi\left(u, \frac{n}{c}\right)\right] & =\sum_{i=0}^{u+n} \operatorname{IF}_{\mathrm{x}, i}(n)-\sum_{k=1}^{n} \operatorname{IF}_{\mathrm{x}, u+k}(k) \tilde{H}_{n-k}(n-k) \\
& -\sum_{k=1}^{n} h_{u+k}(k) \operatorname{IFH}_{\mathrm{x}, n-k}(n-k) .
\end{aligned}
$$

Proof: Taking the derivative of (8) for the contaminated single amount distribution, setting $s=0$ and applying then (6) gives the required result.

One might use also alternative formulas of Picard and Lefèvre (1997) or direct recursive formulas. These formulas and the corresponding schemes are given in Appendix.

During the implementation of the algorithms, one may take care to compute each quantity only once. In particular, since computations of $h_{j}(\tau)$ and $\mathrm{IF}_{\mathrm{x}, j}(\tau)$ involve calculation of $h_{i}(\tau)$ and $\mathrm{IF}_{\mathrm{x}, i}(\tau), i \leq j$, these quantities should be stored and summed at the right time. Notice also that some factors do not depend on perturbation point $x$, which enables us to compute influence functions for a set of values of $x$ in a shorter time.

Some of the above sums may be interpreted as influence functions of quantities like $H_{\text {.(.), }}$, $\tilde{H}$. (.) or $\varphi(0,$.$) . The discussion on the comparison between computation times for these$ three methods can be directly adapted from Rullière and Loisel (2004).

Remark 2 Previous computations of influence functions in propositions 8 and 20 for times $n / c, n \notin \mathbb{N}$ and initial reserves $u \notin \mathbb{N}$ can be done by adaptation of formulas (2.7) and (2.11) in Rullière and Loisel (2004). It will sometimes be necessary to find the initial reserve $u \in \mathbb{R}^{+}$respecting some constraints for ruin probabilities and influence functions, so that an adaptation of previous formulas (given in Appendix) may be useful.

\section{Properties of influence functions associated to ruin probabilities}

In this section, we first show that the influence function of the finite-time ruin probability is non-decreasing, bounded and constant after a certain threshold. This leads us to study the particular properties of the influence function for large contamination points $x$. The situation is quite simple in this case since each claim amount replaced by $x$ will cause ruin. Nevertheless, the event "one claim is replaced by $x$ " is strongly dependent on the number of claims on the considered period. A first approach can consist in studying the risk process given the number of claims, but we consider here the cumulated claim amount process, which is sufficient to determine the probability of ruin. This analysis is particularly relevant for lines of business that may be exposed to catastrophe risk. Let us start with an intuitive, and simple result:

Proposition 9 (Monotonicity of IF) For all $u \geq 0$ and $t>0$,

$$
\operatorname{IF}_{\mathrm{x}}[\psi(u, t)]=-\mathrm{IF}_{\mathrm{x}}[\varphi(u, t)]
$$

is non-decreasing in $x$. 
Proof: For each random path of $R_{t}^{(s, x)}$, for any $x^{\prime}>x$, if $R_{t}^{(s, x)}$ reaches the lower barrier 0 , then a fortiori $R_{t}^{\left(s, x^{\prime}\right)}$ also reaches 0 . It follows that

$$
\psi^{\left(s, x^{\prime}\right)}(u, t) \geq \psi^{(s, x)}(u, t),
$$

and the result holds.

The following results are true for $\tau \in \mathbb{R}$, but when $\tau<0$, usual probabilities have to understood formally. In what follows, we assume without loss of generality that $\tau>0$.

Proposition 10 (IF for $x=0$ )

$$
\mathrm{IF}_{0, y}(\tau)=-\mathrm{P}\left[S_{\frac{\tau}{c}}=y\right]+\sum_{i=1}^{y} \frac{\lambda \tau}{c} \frac{i}{y} \prod_{i} \mathrm{IF}_{0, y-i}(\tau)
$$

Proposition 11 Recall that $h_{j}{ }^{(s, x)}(\tau)=\mathrm{P}\left[S_{\tau / c}{ }^{(s, x)}=j\right]$. For $x>j$, we have

$$
h_{j}^{(s, x)}(\tau)=\exp \left(-\frac{\lambda \tau}{c} s\right) h_{j}(\tau(1-s)), \quad x, j \in \mathbb{N}, x>j .
$$

\section{Proof:}

$$
\mathrm{P}\left[S_{\tau / c}{ }^{(s, x)}=j\right]=\sum_{n=0}^{+\infty} \mathrm{P}\left[N_{\tau / c}^{(s, x)}=n\right] \mathrm{P}\left[W^{(s, x) * n}=j\right] .
$$

Since $x>j$, we have

$$
\mathrm{P}\left[W^{(s, x) * n}=j\right]=(1-s)^{n} \mathrm{P}\left[W^{* n}=j\right] .
$$

Proposition 12 (Aggregate claim amount IF for large $x$ ) For $x>j$, the influence function $\mathrm{IF}_{\mathrm{x}, j}(\tau)$ does not depend on $x$ and is given by

$$
\mathrm{IF}_{\mathrm{x}, j}(\tau)=-\frac{\lambda \tau}{c} h_{j}(\tau)-\lambda \frac{\partial}{\partial \lambda} h_{j}(\tau), \quad x, j \in \mathbb{N}, x>j
$$

Proof: From proposition (11), taking derivatives at $s=0$, and using (6), with eventually $\tau \frac{\partial}{\partial \tau} h_{j}(\tau)=\lambda \frac{\partial}{\partial \lambda} h_{j}(\tau)$.

We also remark that for large $x,(5)$ becomes

$$
\mathrm{IF}_{\mathrm{x}, y}(\tau)=-h_{y}(\tau)+\sum_{i=1}^{y} \frac{\lambda \tau}{c} \frac{i}{y} \Pi_{i} \mathrm{IF}_{\mathrm{x}, y-i}(\tau)
$$

We then check that equation (10) satisfies this last equality using (A.2).

It is rather direct to get the ruin probability influence function from the aggregate claim amount influence function, since we can simplify $\operatorname{IF}_{\mathrm{x}}\left[\varphi\left(u, \frac{n}{c}\right)\right]$ in proposition (8). An interesting link occurs then between sensitivity with respect to parameter $\lambda$ and influence function, as shown in the next propositions. 
Proposition 13 Let $\varphi^{(s, x)}\left(u, \frac{n}{c}, \lambda\right)$ be the probability of ruin for contaminated claim amounts when we assume a Poisson intensity $\lambda$. For $x>u+n$, we have

$$
\varphi^{(s, x)}\left(u, \frac{n}{c}, \lambda\right)=\varphi\left(u, \frac{n}{c}, \lambda(1-s)\right) \exp \left(-\lambda \frac{n}{c} s\right)
$$

Proof: Let $N^{\prime}$ be the number of claims replaced by $x$ before time $n / c$. We see that

$$
\varphi^{(s, x)}\left(u, \frac{n}{c}, \lambda\right)=\mathrm{P}\left[N^{\prime}=0\right] \mathrm{P}\left[T_{u}^{(s, x)}>\frac{n}{c} \mid N^{\prime}=0\right],
$$

when $x>u+n$. Then

$$
\varphi^{(s, x)}\left(u, \frac{n}{c}, \lambda\right)=\sum_{k \in \mathbb{N}} \mathrm{P}\left[N^{\prime}=0 \cap N_{n / c}=k\right] \mathrm{P}\left[T_{u}^{(s, x)}>\frac{n}{c} \mid N^{\prime}=0 \cap N_{n / c}=k\right] .
$$

Denote by $N^{s}$ a Poisson process of intensity $\lambda(1-s)$. Then

$$
\varphi^{(s, x)}\left(u, \frac{n}{c}, \lambda\right)=\sum_{k \in \mathbb{N}} \mathrm{P}\left[N_{n / c}^{s}=k\right] \mathrm{P}\left[T_{u}>\frac{n}{c} \mid N_{n / c}=N_{n / c}^{s} \cap N_{n / c}^{s}=k\right] \exp \left(-\lambda \frac{n}{c} s\right),
$$

and the result follows.

Proposition 14 (ruin probability IF for large $x$ ) For $x>u+n$, we have

$$
\operatorname{IF}_{\mathrm{x}}\left[\varphi\left(u, \frac{n}{c}\right)\right]=-\lambda \frac{\partial}{\partial \lambda} \varphi\left(u, \frac{n}{c}\right)-\lambda \frac{n}{c} \varphi\left(u, \frac{n}{c}\right)
$$

Proof: Plug (10) in (8), and use (8). Another way to get this formula is to differentiate (12) with respect to $s$, and then to consider this derivative at $s=0$.

Derivatives with respect to $\lambda$ may be simplified by using relations (A.1), (A.3) and (3). As an example, we get:

Proposition 15 For $x>j$, the influence function $\operatorname{IF}_{\mathrm{x}, j}(\tau)$ does not depend on $x$ and is given by

$$
\operatorname{IF}_{\mathrm{x}, j}(\tau)=-\frac{\lambda \tau}{c} \mathrm{P}\left[W+S_{\tau / c}=j\right], \quad x, j \in \mathbb{N}, x>j
$$

Proof: This is a consequence of (A.3) and (10).

Another possibility is to check that this equation satisfies (11). Use Panjer's formula for $h_{y}(\tau)$, and develop $\mathrm{P}\left[W+S_{\tau / c}=y\right]$ according to its natural convolution, one may check that

$$
\sum_{i=1}^{y} \Pi_{i} \mathrm{P}\left[S_{\tau / c}=y-i\right]\left(1-\frac{i}{y}\right)=\frac{\lambda \tau}{c} \sum_{i=1}^{y} \frac{i}{y} \Pi_{i} \mathrm{P}\left[W+S_{\tau / c}=y-i\right] .
$$


Expressing $\mathrm{P}\left[W+S_{\tau / c}=y-i\right]$ as a natural convolution sum, the result is obtained with a mere sum inversion, checking then that one sum can be suppressed thanks to Panjer's formula.

Proposition 16 For $x>n$, the influence function of the non-ruin probability without initial reserve does not depend on $x$ and is given by

$$
\begin{aligned}
\operatorname{IF}_{\mathrm{x}}\left[\varphi\left(0, \frac{n}{c}\right)\right] & =-\frac{\lambda n}{c} \mathrm{E}\left[\left(1-\frac{W+S_{n / c}}{n}\right)_{+}\right] \\
& =-\frac{\lambda n}{c} \mathrm{P}\left[W+S_{n / c} \leq n\right]+\frac{\lambda}{c} \sum_{j=0}^{n} j \mathrm{P}\left[W+S_{n / c}=j\right] .
\end{aligned}
$$

Proof: Plug (14) in the expression of $\varphi\left(0, \frac{n}{c}\right)$ as given in (7).

Remark 3 For $x>u+n$, the influence function of the non-ruin probability without initial reserve does not depend on $x$ and is given by non-recursive sums involving only distributions of $S_{t}$ and of $W$. Since these expressions are quite long, they are not given here. They may be obtained by direct insertion of equation (14) into, for example, Proposition 8.

\section{Weak convergence of finite-ruin probabilities based on empirical distribu- tion}

In this section we show that the rescaled empirical finite-time non-ruin probability starting from zero converges in distribution to a Gaussian distribution. To this end, let us consider the empirical finite-time non-ruin probability with zero initial reserve and within time horizon $t>0$

$$
\varphi^{N}(0, t)=P\left(\exists s \in[0, t], \quad c t-\sum_{j=1}^{N_{t}} Y_{j}^{N}<0\right)
$$

where the $\left(Y_{j}^{N}\right)_{j \geq 1}$ are i.i.d. random variables drawn from the empirical distribution of a random sample $\left\{\bar{Y}_{1}, \ldots, Y_{N}\right\}$ of size $N \geq 1$ from the distribution of $W$. Given that

$$
\varphi(0, t)=\frac{1}{c t} E\left[\left(c t-\sum_{j=1}^{N_{t}} W_{j}\right)_{+}\right]
$$

and

$$
\varphi^{N}(0, t)=\frac{1}{c t} E\left[\left(c t-\sum_{j=1}^{N_{t}} Y_{j}^{N}\right)_{+}\right]
$$

we may rewrite the difference between the finite-time non-ruin probability and its estimate based on the empirical distribution of $W$ as

$$
\varphi(0, t)-\varphi^{N}(0, t)=\sum_{k \geq 1} \mathrm{P}\left[N_{t}=k\right]\left[E\left[\frac{1}{c t}\left(c t-\sum_{j=1}^{k} W_{j}\right)_{+}\right]-\frac{1}{N^{k}} \sum_{1 \leq i_{1}, \ldots, i_{k} \leq N} \Phi_{k}\left(Y_{i_{1}}, \ldots, Y_{i_{k}}\right)\right],
$$


where, for $k \geq 1$, and for $y_{1}, \ldots, y_{k} \in \mathbb{R}$, we set

$$
\Phi_{k}\left(y_{1}, \ldots, y_{n}\right)=\mathrm{E}\left[\frac{1}{c t}\left(c t-\sum_{j=1}^{k} y_{j}\right)_{+}\right] \text {. }
$$

One recognizes a typical von Mises functional, closely related to U-statistics, for which many asymptotic results are known (see for example Hoeffding (1948), Von Mises (1947) and Gotze (1984)).

Let $\varphi_{k}(0, t)=\mathrm{E}\left[\frac{1}{c t}\left(c t-\sum_{j=1}^{k} W_{j}\right)_{+}\right], k \geq 1$, with $\varphi_{0}(0, t)=1$, and consider the process

$$
\xi_{k}^{N}=\mathrm{P}\left[N_{t}=k\right]\left[\varphi_{k}(0, t)-\frac{1}{N^{k}} \sum_{1 \leq i_{1}, \ldots, i_{k} \leq N} \Phi_{k}\left(Y_{i_{1}}, \ldots, Y_{i_{k}}\right)\right], k, N \geq 1 .
$$

We get thus a sequence $\left(\zeta_{N}\right)_{N \geq 1}$ taking values in the Banach space $l^{2}\left(\mathbb{R}^{+\infty}\right)$, where for all $N \geq 1, \zeta_{N}$ is defined as

$$
\zeta_{N}=\left(\xi_{k}^{N}\right)_{k \geq 1}
$$

which induces a measure on the space $\mathbb{R}^{+\infty}$. From Theorem 7.4 of Hoeffding (1948), the finite-dimensional projections weakly converge to a Gaussian distribution:

$$
\forall K_{0} \geq 1, \quad \sqrt{N}\left(\xi_{1}^{N}, \ldots, \xi_{K_{0}}^{N}\right) \rightarrow Z_{K_{0}} \text { in distribution as } N \rightarrow+\infty
$$

where for $K_{0} \geq 1, Z_{K_{0}}$ follows a Gaussian distribution with mean vector

$$
0_{K_{0}}=(0, \ldots, 0)
$$

and the $K_{0} \times K_{0}$ covariance matrix $\Gamma_{K_{0}}$ defined for $1 \leq i, j \leq K_{0}$ by

$$
\left(\Gamma_{K_{0}}\right)_{i j}=i j \mathrm{P}\left[N_{t}=i\right] \mathrm{P}\left[N_{t}=j\right] \mathrm{E}\left[\left(\varphi_{i-1}^{(Y)}-\varphi_{i}\right)\left(\varphi_{j-1}^{(Y)}-\varphi_{j}\right)\right]
$$

with $\varphi_{k}^{(x)}(0, t)=\mathrm{E}\left[\frac{1}{c t}\left(c t-x-S_{t}\right)_{+} \mid N_{t}=k\right]$, and $\varphi^{(x)}(0, t)=\mathrm{E}\left[\frac{1}{c t}\left(c t-x-S_{t}\right)_{+}\right]$(we omit the argument $(0, t)$ for more clarity).

Notice that for claim amounts taking values in

$$
\delta \mathbb{N}=\{0, \delta, 2 \delta, \ldots\},
$$

where $\delta>0$ and $t>0$ are fixed, we can assume without restriction that claim amounts take values in $\{\delta, 2 \delta, \ldots\}$ (just change the intensity $\lambda$ into $\lambda(1-P(W=0))$ and $P(W=$ $k \delta$ ) into

$$
\frac{P(W=k \delta)}{1-P(W=0)}
$$

for $k \geq 1$ ). Then, if

$$
N_{t}>\left\lfloor\frac{c t}{\delta}\right\rfloor
$$


ruin is certain since we have at least

$$
\left\lfloor\frac{c t}{\delta}\right\rfloor+1
$$

jumps of size greater or equal to $\delta$. This is true both for empirical and "true" distributions of $W$. Therefore, for all $N \geq 1$,

$$
\xi_{k}^{N}=0
$$

for all $k \geq K+1$, where

$$
K=\left\lfloor\frac{c t}{\delta}\right\rfloor
$$

Theorem 1 If claim amounts take values in $\delta \mathbb{N} \backslash\{0\}$,

$$
\sqrt{N}\left(\varphi(0, t)-\varphi^{N}(0, t)\right) \rightarrow Z \text { in distribution as } N \rightarrow+\infty,
$$

where $Z \sim \mathcal{N}\left(0, V_{0}\right)$, with variance

$$
V_{0}=V_{Y}\left[\lambda t \varphi^{(Y)}(0, t)\right]=V_{Y}\left[\operatorname{IF}_{Y}[\varphi(0, t)]\right]
$$

with $\varphi^{(x)}(0, t)=\mathrm{E}\left[\frac{1}{c t}\left(c t-x-S_{t}\right)_{+}\right], x \in \mathbb{N}$, and where $Y$ is a r.v. distributed as $W$.

Remark 4 Notice that the identity between variances given in Theorem 1 corresponds to the general relation between asymptotic variances and influence functions given in (4).

Remark 5 This theorem is only valid for $u=0$. We leave the theoretical proof of the general case $u>0$ for future research, but provide a kind of computer-aided proof in the numerical analysis section to show that the methods we propose to compute the Estimation Risk Solvency Margin (to be defined in section 6.1) are implementable. The case $u>0$ is important for applications to Estimation Risk Solvency Margin (see section 6.1).

Proof: Theorem 7.4 of Hoeffding (1948) yields that the limiting variance is given by

$$
V_{0}=\sum_{i=1}^{K} \sum_{j=1}^{K}\left(\Gamma_{K_{0}}\right)_{i j}
$$

Hence

$$
V_{0}=v_{1}-v_{2},
$$

with

$$
v_{1}=E_{Y}\left[\sum_{i=1}^{K} i \mathrm{P}\left[N_{t}=i\right] \varphi_{i-1}^{(Y)} \sum_{j=1}^{K} j \mathrm{P}\left[N_{t}=j\right] \varphi_{j-1}^{(Y)}\right]
$$

and

$$
v_{2}=\sum_{i=1}^{K} \sum_{j=1}^{K} i j \mathrm{P}\left[N_{t}=i\right] \mathrm{P}\left[N_{t}=j\right] \varphi_{i}(0, t) \varphi_{j}(0, t) .
$$

Using the identities

$$
i \mathrm{P}\left[N_{t}=i\right]=\lambda t \mathrm{P}\left[N_{t}=i-1\right]
$$

and

$$
\varphi_{k}^{(Y)}=0, \quad k>K
$$


one obtains that

$$
v_{1}=(\lambda t)^{2} E_{Y}\left[\sum_{i=0}^{\infty} \mathrm{P}\left[N_{t}=i\right] \varphi_{i}^{(Y)} \sum_{j=0}^{\infty} j \mathrm{P}\left[N_{t}=j\right] \varphi_{j}^{(Y)}\right]
$$

so that

We can show that

$$
v_{1}=(\lambda t)^{2} E_{Y}\left[\left(\varphi^{(Y)}(0, t)\right)^{2}\right]
$$

Using the above arguments, one gets that

$$
\varphi_{i}=E_{Y}\left[\varphi_{i-1}^{(Y)}\right]
$$

$$
v_{2}=(\lambda t)^{2} \sum_{i=0}^{\infty} \mathrm{P}\left[N_{t}=i\right] E_{Y}\left[\varphi_{i}^{(Y)}(0, t)\right] \sum_{j=0}^{\infty} \mathrm{P}\left[N_{t}=j\right] E_{Y}\left[\varphi_{j}^{(Y)}(0, t)\right]
$$

and therefore

$$
v_{2}=(\lambda t)^{2}\left(E_{Y}\left[\varphi^{(Y)}(0, t)\right]\right)^{2}
$$

We next consider the last identity (20)

$$
V_{Y}\left[\lambda t \varphi^{(Y)}(0, t)\right]=V_{Y}\left[\operatorname{IF}_{Y}[\varphi(0, t)]\right]
$$

Given $0<s<1$, let $\varepsilon$ be a generic Bernoulli random variable with $P(\varepsilon=1)=s=$ $1-P(\varepsilon=0)$. Then the random variable $\varepsilon y+(1-\varepsilon) W$ has $F^{(s, y)}(u)$ as a distribution function. One must thus consider the following limit,

$$
\lim _{s \rightarrow 0} \frac{1}{s}\left(E_{(\varepsilon, W)}\left[\left(c t-\sum_{i=1}^{k}\left(\varepsilon_{i} y+\left(1-\varepsilon_{i}\right) W_{i}\right)_{+}\right]-E_{W}\left[\left(c t-\sum_{i=1}^{k} W_{i}\right)_{+}\right]\right) .\right.
$$

Using independence and Fubini's Theorem, one is led to consider first the integral over $\varepsilon$ given $W$

$$
I_{w}:=E_{\varepsilon}\left[\left(c t-y \sum_{i=1}^{k} \varepsilon_{i}-\sum_{i=1}^{k} W_{i}+\sum_{i=1}^{k} \varepsilon_{i} W_{i}\right)_{+}\right] .
$$

The collection of i.i.d. random variables $\left(\varepsilon_{1}, \varepsilon_{2}, \cdots, \varepsilon_{k}\right)$ can be seen as corresponding to random subsets $J$ of $\{1,2, \cdots, k\}$, of law $P(J)=s^{|J|}(1-s)^{k-|J|}$, where $|J|$ denotes the size of $J$ with $|J|=\sum_{i=1}^{k} \varepsilon_{i}$. Then we can write

$$
I_{w}=\sum_{n=0}^{k} s^{|J|}(1-s)^{k-|J|} \sum_{|J|=n}\left(c t-y n-\sum_{i=1}^{k} W_{i}+\sum_{i \in J} \varepsilon_{i} W_{i}\right)_{+} .
$$

We shall see that only the first two terms corresponding to $n=0$ and $n=1$ contribute to the limit: one first check the behavior of

$$
\lim _{s \rightarrow 0} \frac{E_{W}\left[I_{w}-\tilde{I}_{w}\right]}{s}
$$

where we set $\tilde{I}_{w}=\left(c t-\sum_{i=1}^{k} W_{i}\right)_{+}$. Let $I_{w}=I_{w}^{0}+I_{w}^{1}$, where

$$
I_{w}^{0}=(1-s)^{k}\left(c t-\sum_{i=1}^{k} W_{i}\right)_{+} \text {and } I_{w}^{1}=s(1-s)^{k-1} \sum_{j=1}^{k}\left(c t-y-\sum_{i \neq j} W_{i}\right)_{+} .
$$


Using the fact that $\left.\left((1-s)^{k}-1\right) / s\right) \sim-k$ as $s \rightarrow 0$, one gets the equivalent expression

$$
-k E_{W}\left[\left(c t-\sum_{i=1}^{k} W_{i}\right)_{+}\right]+k(1-s)^{k-1} E_{W}\left[\left(c t-y-\sum_{i=1}^{k-1} W_{i}\right)_{+}\right] \text {. }
$$

The next step consists in taking the variance of the above random variable when $Y$ is distributed like $W$, and is independent of the $W_{i}$. The first term is constant, and therefore the variance is given by, collecting the terms related to $k$,

$$
\begin{gathered}
\mathrm{V}_{Y}\left[\sum_{k \geq 0} \frac{(\lambda t)^{k}}{k !} e^{-\lambda t} k E_{W}\left[\left(c t-Y-\sum_{i=1}^{k-1} W_{i}\right)_{+}\right]\right] \\
=\mathrm{V}_{Y}\left[\lambda t \sum_{k \geq 0} \frac{(\lambda t)^{k}}{k !} e^{-\lambda t} E_{W}\left[\left(c t-Y-\sum_{i=1}^{k} W_{i}\right)_{+}\right]\right] \\
=\mathrm{V}_{Y}\left[\lambda t \varphi^{(Y)}(0, t)\right],
\end{gathered}
$$

which corresponds to the required identity. It remains to check that the terms related to $n \geq 2$ do not contribute to the limit $s \rightarrow 0$. This follows from bounded convergence.

In the case where claim amounts follow a continuous distribution $F_{W}$, we can approximate $F_{W}$ with a sequence $\left(F_{W_{p}}\right)_{p \geq 1}$ of discretized versions of $F_{W}$ such that $W_{p}$ takes values in $\frac{1}{p} \mathbb{N}$ for $p \geq 1$, in the sense that for all $x \in \mathbb{R}$,

$$
F_{W_{p}}(x) \rightarrow F_{W}(x)
$$

as $p$ tends to infinity. Denote respectively by $\varphi_{p}(0, t)$ and $\varphi_{p}^{N}(0, t)$ the finite-time ruin probability with claim amount distribution $F_{W_{p}}$ and the related empirical version. Clearly, for a fixed $N \geq 1$,

converges in distribution to

$$
\left(\sqrt{n}\left[\varphi_{p}(0, t)-\varphi_{p}^{n}(0, t)\right]\right)_{1 \leq n \leq N}
$$

$$
\left(\sqrt{n}\left[\varphi(0, t)-\varphi^{n}(0, t)\right]\right)_{1 \leq n \leq N}
$$

as $p \rightarrow+\infty$. As the weak convergence of a family of measures on the Banach space $l^{2}\left(\mathbb{R}^{+\infty}\right)$ is ensured by the weak convergence of the finite-dimensional projections (see for example Billingsley (1999), chapter 1.5), we get that

$$
\left(\sqrt{N}\left[\varphi_{p}(0, t)-\varphi_{p}^{N}(0, t)\right]\right)_{N \geq 1}
$$

converges weakly toward

$$
\left(\sqrt{N}\left[\varphi(0, t)-\varphi^{N}(0, t)\right]\right)_{N \geq 1},
$$

as $p \rightarrow+\infty$. For given $p \geq 1$, Theorem 1 yields that

$$
\sqrt{N}\left(\varphi_{p}(0, t)-\varphi_{p}^{N}(0, t)\right)
$$


converges in distribution to

$$
Z_{p} \sim \mathcal{N}\left(0, \sigma_{p}^{2}\right)
$$

where

$$
\sigma_{p}^{2}=V_{W_{p}}\left[\lambda t \varphi_{p}^{\left(W_{p}\right)}(0, t)\right]
$$

and

$$
\varphi_{p}^{(x)}(0, t)=\mathrm{E}\left[\frac{1}{c t}\left(c t-x-S_{t}^{p}\right)_{+}\right] .
$$

Here, $S_{t}^{p}$ corresponds to the cumulated claim amount up to time $t$ for individual claim amounts distributed as $W_{p}$. As

$$
\sigma_{p}^{2}=V_{W_{p}}\left[\lambda t \varphi_{p}^{\left(W_{p}\right)}(0, t)\right] \rightarrow V_{W}\left[\lambda t \varphi^{(W)}(0, t)\right], p \rightarrow \infty
$$

$Z_{p}$ converges in distribution to

$$
Z \sim \mathcal{N}\left(0, \sigma^{2}\right)
$$

with

$$
\sigma^{2}=\lim _{p \rightarrow+\infty} \sigma_{p}^{2}=V_{W}\left[\lambda t \varphi^{(W)}(0, t)\right]
$$

Hence

$$
\sqrt{N}\left(\varphi(0, t)-\varphi^{N}(0, t)\right) \rightarrow Z \text { in distribution as } N \rightarrow+\infty,
$$

with the following commutative diagram :

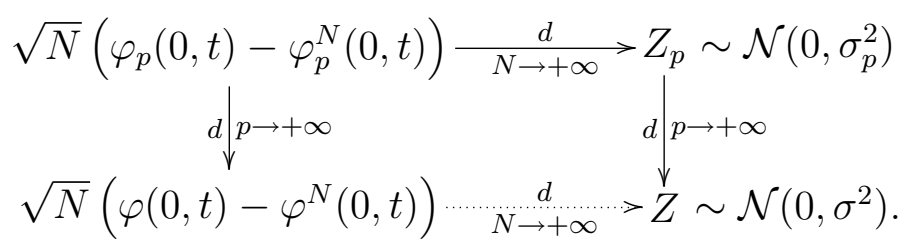

Remark 6 Note that as the infinite-time ruin probability starting from zero only depends on the claim size distribution through its expected value, the asymptotic variance of

$$
\sqrt{N}\left(\varphi(0, t)-\varphi^{N}(0, t)\right)
$$

tends to the asymptotic variance of

$$
\sqrt{N}\left(E\left[W_{1}\right]-\mu_{W}^{N}\right)
$$

multiplied by $\lambda^{2} / c^{2}$ as $t$ goes to infinity, where $\mu_{W}^{N}$ is the (random) empirical average of $W_{1}$ obtained from an $N$-sample of the claim size distribution. From the central limit theorem and from the same way of reasoning as above, the asymptotic variance of

$$
\sqrt{N}\left(\varphi(0, t)-\varphi^{N}(0, t)\right)
$$

converges to

$$
\frac{\lambda^{2}}{c^{2}} \operatorname{Var}\left(W_{1}\right)
$$

as $t$ tends to $+\infty$. 


\section{Some applications of influence functions}

\subsection{Reliable finite-time ruin probabilities}

Recall that $\psi(u, t)$ denotes the finite-time ruin probability, which is seen as a functional of the claim amount distribution $F$. Similarly, $\psi^{N}(u, t)$ denotes the random finite-time ruin probability, with claim amounts drawn from the empirical distribution $F_{N}$ associated with an i.i.d. sample of distribution $F$.

Definition 2 The reliable finite-time ruin probability $\psi_{1-\varepsilon}^{N \text {,reliable }}(u, t)$ is the $(1-\varepsilon)$-quantile of the (random) bootstrapped finite-time ruin probability $\psi^{N}(u, t)$ :

$$
\psi_{1-\varepsilon}^{N, r e l i a b l e}(u, t)=\inf _{s \geq 0}\left\{\mathrm{P}\left[\psi^{N}(u, t) \geq s\right] \leq \varepsilon\right\} .
$$

We checked in the previous sections that $\psi^{N}(u, t)$ can be approximated for large claim size databases (see section 7.2 to know what large $N$ means in practice) by a Gaussian random variable of mean $\psi(u, t)$ when $u=0$. Numerical simulations seem to confirm the asymptotic Normality of $\psi^{N}(u, t)$, for arbitrary $u$ (see Section 7.1). When $\psi^{N}(u, t)$ is approximately Gaussian of mean $\psi(u, t)$ and variance $V_{u} / N$, one can consider the approximation

$$
\tilde{\psi}_{1-\epsilon}^{N, \text { reliable }}(u, t)=\psi(u, t)+\frac{\sqrt{V_{u}}}{\sqrt{N}} \Phi^{-1}(1-\epsilon)
$$

where $\Phi$ denotes the distribution function of a standard Normal r.v., and where $V_{u}$ is the asymptotic variance of $\sqrt{N}\left(\varphi(u, t)-\varphi^{N}(u, t)\right)$ :

$$
V_{u}=V_{Y}\left[\operatorname{IF}_{Y}[\varphi(u, t)]\right], u \geq 0,
$$

which can be obtained from Sections 3 and 5. Section 7 gives examples where the computation time required to estimate the variance is reasonable, but the computation time can heavily increase when discretization step $\delta$ becomes smaller.

One judicious choice is $\varepsilon=2.5 \%$, as the 97.5 percentile of a Gaussian $\left(\mu, \sigma^{2}\right)$ random variable can be approximated by $\mu+2 \sigma$. In this case, one gets the pragmatic approximation $\Phi^{-1}(1-\epsilon) \simeq 2$.

If $u_{\eta}$ and $u_{\eta, \varepsilon}$ are respectively defined as the initial capital required to ensure that

$$
\psi\left(u_{\eta}, t\right) \leq \eta
$$

and

$$
\psi_{1-\varepsilon}^{N, \text { reliable }}\left(u_{\eta, \varepsilon}, t\right) \leq \eta,
$$

the Estimation Risk Solvency Capital $E R S M_{\eta, 1-\varepsilon}$ can be defined as the additional capital needed to take estimation risk into account :

$$
E R S M_{\eta, 1-\varepsilon}=u_{\eta, 1-\varepsilon}-u_{\eta}
$$

which can be obtained from the results of Section 7 . It might be thus interesting to determine solvency requirements from $\psi_{97.5 \%}^{N \text {,reliable }} \leq \eta$ with $1-\eta<99.5 \%$, rather than from 
$\psi \leq 1-99.5 \%$. This might lead to a gain in robustness, as $99.5 \%$ safety levels are almost impossible to handle in practice. We give examples of values of $\eta$ that lead to values of $u_{\eta, 97.5 \%}$ of the same magnitude as $u_{1-99.5 \%}$ in section 7.2 .

In practical cases, one may ignore the exact distribution $F$. If the claim size database contains $N \geq 1$ observed claim amounts $O_{N}=\left\{w_{1}, \ldots, w_{N}\right\}$, then estimators of $\psi(u, t)$, $\psi_{1-\varepsilon}^{N \text {,reliable }}(u, t)$ and $\tilde{\psi}_{1-\epsilon}^{N \text {,reliable }}(u, t)$ may be obtained, for example, by respective plug-in estimators, $\psi^{O_{N}}(u, t), \psi_{1-\varepsilon}^{O_{N} \text {,reliable }}(u, t)$ and $\tilde{\psi}_{1-\epsilon}^{O_{N} \text {,reliable }}(u, t)$, when $F$ is replaced by the empirical distribution function $F_{O_{N}}$ from $O_{N}$. These estimators may also suffer from estimation risk. From Propositions 5, 6, 7 and 8, and with the same kind of reasoning as in Sections 3 and 4 , it can be shown that the influence function of the influence function of the ruinprobability is bounded. From (4) and from Proposition 15, we obtain that estimators $\psi^{O_{N}}(u, t), \psi_{1-\varepsilon}^{O_{N}, \text { reliable }}(u, t)$ and $\tilde{\psi}_{1-\epsilon}^{O_{N} \text {,reliable }}(u, t)$ are robust according to Hampel's definition, as their influence functions are bounded.

\subsection{Catastrophe claim contamination}

For infinite-time ruin probabilities, in the case of heavy-tailed claim amount distributions, it would be better to use some methods from the theory of extremes. However, in the case of finite-time ruin probabilities, we want to point out here a very simple relation that gives the impact of the contamination of data by large claim amounts. Assume that the solvency capital requirements of an insurance company are determined in such a way that the finite-time ruin probability is less than $\varepsilon$. For some lines of business exposed to catastrophe risk, the following question arises: if the risk corresponding to claims that are larger than a given deterministic amount $M>0$ are transferred using reinsurance or securitization, what is the effect of this transfer on the ruin probability? Is the decrease of the required capital level enough to finance this risk transfer in order to maintain the same premium income rate? Is it possible to determine easily the given amount $M$ necessary to get a given level of ruin probability?

Consider the truncated random variable $\tilde{W}$ such that $\mathrm{P}[\tilde{W}=x]=\mathrm{P}[W=x], \quad 0<x<$ $M$ and $\mathrm{P}[\tilde{W}=0]=\mathrm{P}[W \geq M]$. Recall that $\mathrm{P}[W=0]=0$. Let $\left(\tilde{N}_{t}\right)_{t \geq 0}$ be the Poisson process (with intensity $\lambda \mathrm{P}[W>M]$ ) defined for $t \geq 0$ by

$$
\tilde{N}_{t}=\sum_{k \geq 1} \mathbf{1}_{T_{i} \leq t} \mathbf{1}_{W_{i}>M}
$$

where $\left(T_{i}\right)_{i \geq 1}$ is the sequence of jump instants of $\left(N_{t}\right)_{t \geq 0} . \tilde{N}_{t}$ represents of course the number of claims of size larger than $M$ up to time $t \geq 0$. Denote by $\tilde{\varphi}(u, t)$ the finite-time non-ruin probability in the modified model.

From the total probability formula, the classical finite-time non-ruin probability satisfies the following equation: for all $u, M$ and $t \geq 0$, 


$$
\begin{aligned}
\varphi(u, t) & =\mathrm{P}\left[\forall s \leq t, u+c t-S_{t} \geq 0 \mid \tilde{N}_{t}=0\right] \cdot \mathrm{P}\left[\tilde{N}_{t}=0\right] \\
& +\mathrm{P}\left[\forall s \leq t, u+c t-S_{t} \geq 0 \mid \tilde{N}_{t}>0\right] \cdot \mathrm{P}\left[\tilde{N}_{t}>0\right]
\end{aligned}
$$

As $\left(\tilde{N}_{t}\right)_{t \geq 0}$ and $\left(N_{t}-\tilde{N}_{t}\right)_{t \geq 0}$ are two independent Poisson processes,

$$
\mathrm{P}\left[\forall s \leq t, u+c t-S_{t} \geq 0 \mid \tilde{N}_{t}=0\right]
$$

is exactly $\tilde{\varphi}(u, t)$. If besides $M>c t$, then any claim of size larger than $M$ causes ruin, and consequently

As

$$
\mathrm{P}\left[\forall s \leq t, u+c t-S_{t} \geq 0 \mid \tilde{N}_{t}>0\right]=0 .
$$

$$
\mathrm{P}\left[\tilde{N}_{t}=0\right]=e^{-\lambda \mathrm{P}[W>M] t}
$$

equation (22) simplifies for $M>c t$ into

$$
\varphi(u, t)=\tilde{\varphi}(u, t) e^{-\lambda \mathrm{P}[W>M] t} .
$$

If follows that when it makes sense, determining the minimal value $M_{0}$ of $M>c t$ such that $\tilde{\varphi}(u, t) \geq 1-\epsilon$ is straightforward since

$$
\mathrm{P}[W>M]=\frac{1}{\lambda t} \ln \left[\frac{\tilde{\varphi}(u, t)}{\varphi(u, t)}\right] .
$$

This leads to the following condition :

$$
\mathrm{P}[W>M] \leq \frac{1}{\lambda t} \ln \left[\frac{1-\epsilon}{\varphi(u, t)}\right],
$$

and so $M$ has to be greater than

$$
M_{0}=\operatorname{VaR}_{\alpha}(W),
$$

where the Value-at-Risk level $\alpha$ is given by

$$
\alpha=\frac{1}{\lambda t} \ln \left[\frac{1-\epsilon}{\varphi(u, t)}\right] .
$$

Equation (23) may also be used to evaluate the influence of large claims and the impact of an underestimation of catastrophe risk.

\section{$7 \quad$ Numerical examples}

In this section, we first show how the influence function of finite-time ruin probabilities may look like. Then we analyze the impact of the size of the claim size database on the asymptotic variance of the estimator of finite-time ruin probabilities and thus on the estimation-risk solvency margin (ERSM), obtained from difference between the reserves that are needed to control the reliable finite-time ruin probability (whose definition and main properties were given in subsection 6.1) and the ones needed to control the empirical finite-time ruin probability. 


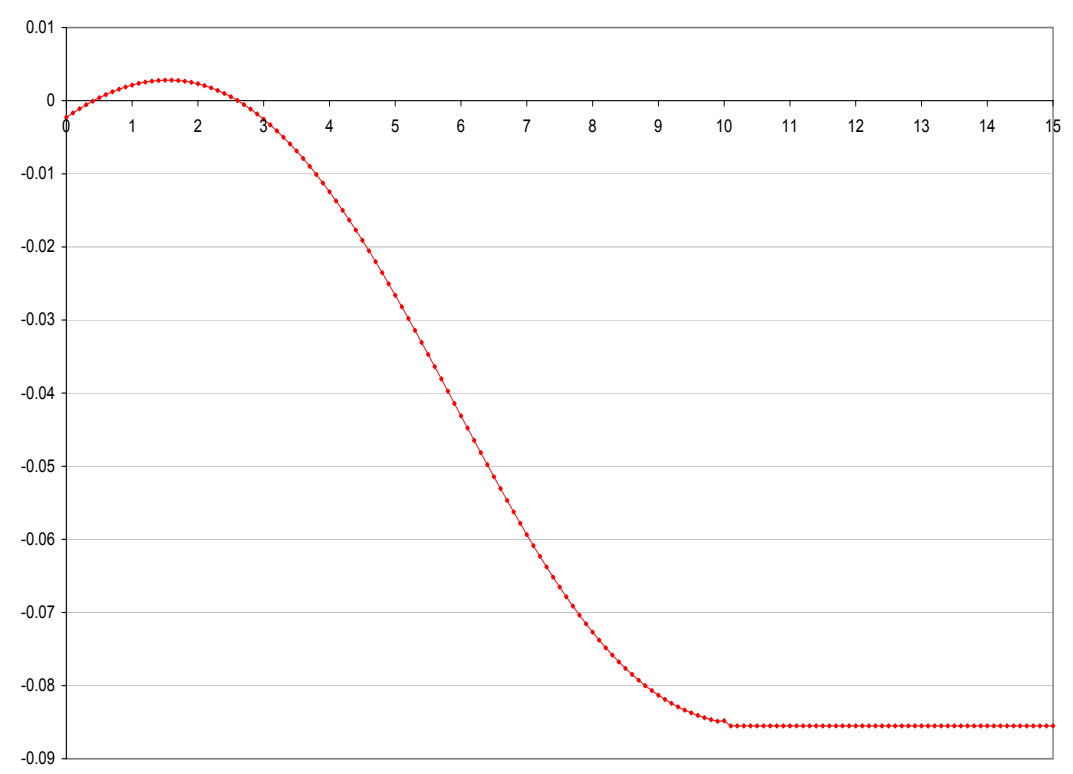

Figure 1. Aspect of the influence function $\operatorname{IF}_{\mathrm{x}}\left[\mathrm{P}\left[S_{t}=u\right]\right]=\mathrm{IF}_{\mathrm{x}, u}(c t)$ as a function of $x$, for $\lambda_{0}=1, c=1.1, t=10$, for discrete exponentially distributed claim amounts with $\delta=0.1$.

\subsection{Numerical analysis of influence functions}

The results presented hereafter have been obtained for parameters $\lambda_{0}=1, c=1.1$, and $t=10$.

We first consider the case where $W_{0}$ is exponentially distributed with parameter 1 . We then define the distribution function $F_{\delta}$ of a discrete claim amount $W_{\delta}$ with $F_{\delta}(i \delta)$ defined on each interval $[i \delta, i \delta+\delta[$, such that

$$
F_{\delta}(i \delta)=\frac{1}{\delta} \int_{[i \delta, i \delta+\delta[} F_{W_{0}}(x) d x
$$

In order to cancel $\pi_{0}=\mathrm{P}\left[W_{\delta}=0\right]$, the Poisson parameter $\lambda_{0}$ has been modified into $\lambda=\lambda_{0}\left(1-\pi_{0}\right)$, and the $\pi_{i}$ have been changed into $\mathrm{P}[W=i]=\pi_{i} /\left(1-\pi_{0}\right)$. All amounts $(c, u, W)$ are expressed in $\delta$ money unit, in order to get integer-valued amounts. This discretization procedure is fully described in De Vylder (1999).

The interest of such a distribution is that some results for continuous exponential claims distribution may be obtained as $\delta$ tends to 0 .

Consider first the influence function of the probability that the aggregate claim amount reaches a value $u$ at time $t$. Figure 1 illustrates the non-monotonicity of this function. In this particular example, changing some claim amounts into some of value 1 or 2 may increase the probability that the aggregate claim amount is 10 . For other values, like 5, it may decrease this probability. For perturbation points $x>u$, the influence function is obviously unchanged since changing only one claim into $x$ implies that $S_{t}$ will not reach $u$. 


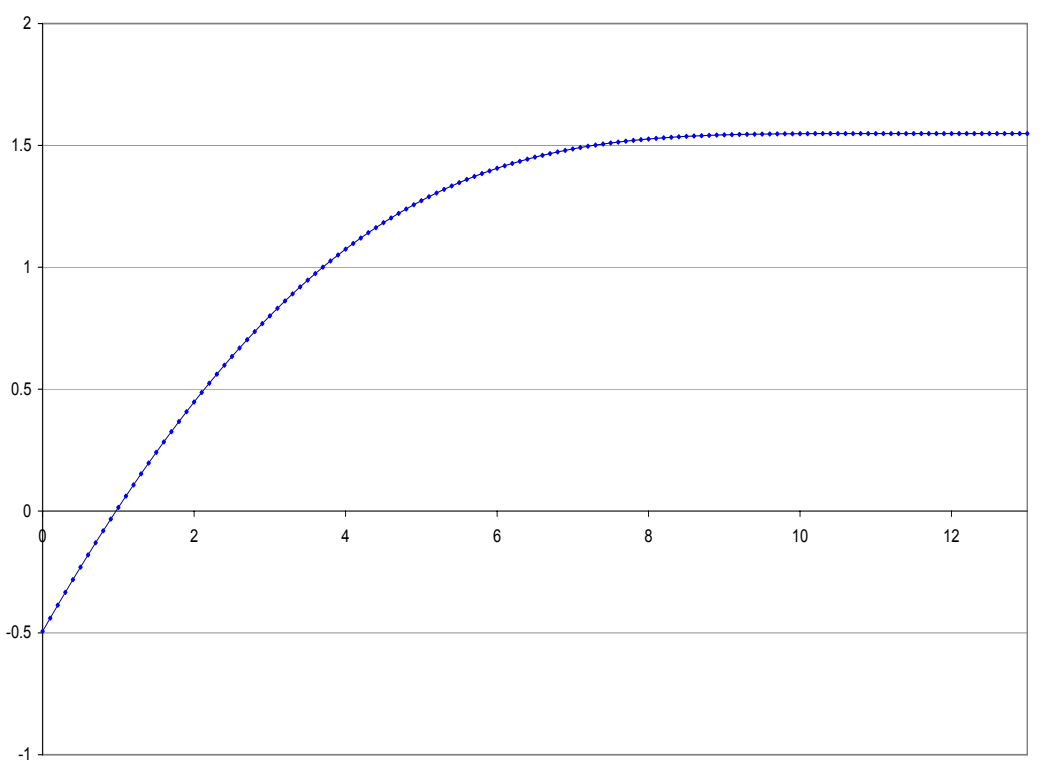

Figure 2. Aspect of the influence function $\operatorname{IF}_{\mathrm{x}}[\psi(0, t)]$ as a function of $x$, for $\lambda_{0}=1, c=1.1, t=10$, for discrete exponentially distributed claim amounts with $\delta=0.1$.

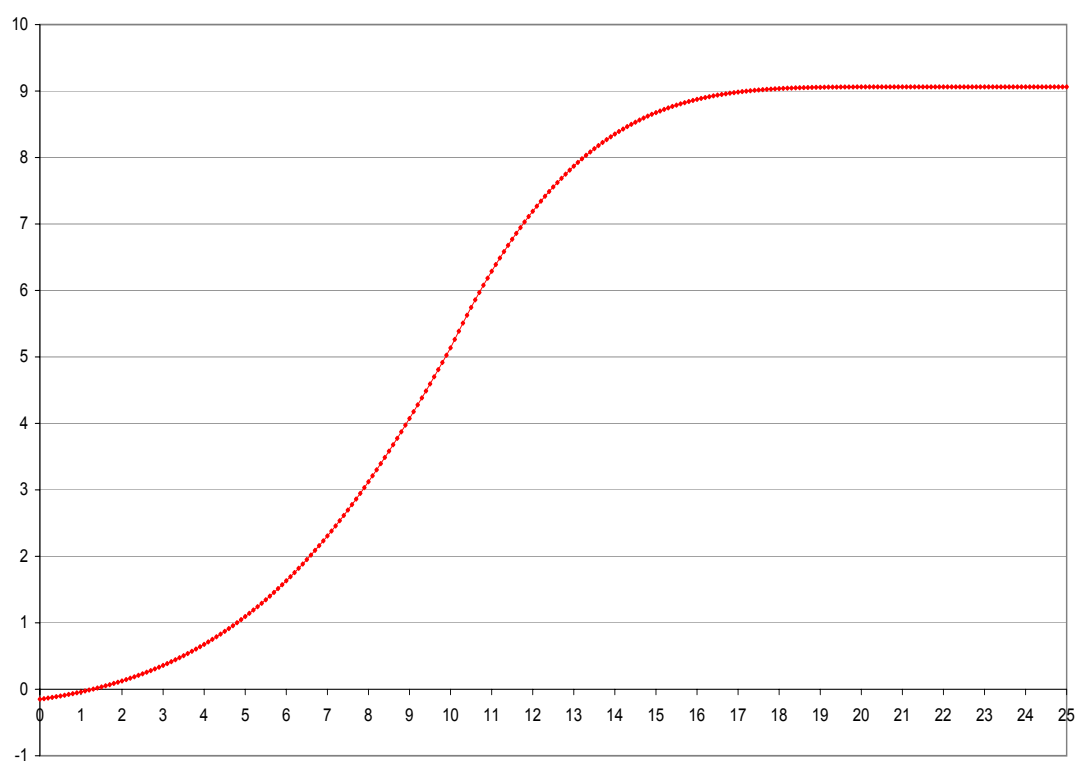

Figure 3. Aspect of the influence function $\operatorname{IF}_{\mathrm{x}}[\psi(u, t)]$ as a function of $x$, for $\lambda_{0}=1, c=1.1, t=10, u=10$, for discrete exponentially distributed claim amounts with $\delta=0.1$.

We have checked numerically that (see equation (14)):

$$
\mathrm{IF}_{\mathrm{x}, j}(\tau)=-\frac{\lambda \tau}{c} \mathrm{P}\left[W+S_{\tau / c}=u\right], \quad x, j \in \mathbb{N}, x>j
$$

In this special case, where $\lambda_{0}=1, c=1.1, t=10, u=10$, for discrete exponential claims with $\delta=0.1$, with standard, 64-bit arithmetic precision, $\mathrm{P}\left[W+S_{t}=u\right]=$ 0.00898542154104457 and $\mathrm{IF}_{\mathrm{x}, u}(c t)=-0.0855075913881109$.

From recursive schemes given in proposition 8 and 20, which give similar results, we draw the influence function of ruin probability as a function of the contamination point $x$. 
We get as an example in Figures 2 and 3 the shape of the influence function of the ruin probability within finite time $\operatorname{IF}_{\mathrm{x}}[\psi(0, t)]$ and $\operatorname{IF}_{\mathrm{x}}[\psi(u, t)]$. We can verify that this influence function starts at a given negative value, is non-decreasing, bounded and constant for $x>u+c t$.

We have checked numerically that (see proposition 16):

$$
\operatorname{IF}_{\mathrm{x}}\left[\varphi\left(0, \frac{n}{c}\right)\right]=-\frac{\lambda n}{c} \mathrm{E}\left[\left(1-\frac{W+S_{n / c}}{n}\right)_{+}\right] .
$$

In this special case where $\lambda_{0}=1, c=1.1, t=10, u=10$, for discrete exponentially distributed claim amounts with $\delta=0.1$, with standard 64-bit arithmetic precision,

$$
\mathrm{E}\left[\left(1-\frac{W+S_{t}}{c t}\right)_{+}\right]=0.15912803689065 \text { and } \operatorname{IF}_{\mathrm{x}}[\varphi(0, t)]=-1.51430348533833 .
$$

We also checked in this case that numerically (see proposition 7) $\operatorname{IF}_{\mathrm{x}}\left[\varphi\left(0, \frac{n}{c}\right)\right]=\sum_{j=0}^{n} \frac{n-j}{n} \operatorname{IF}_{\mathrm{x}, j}(n)$.

At last, we checked that we retrieve numerically for small values of $s$ :

$$
\begin{aligned}
\operatorname{IF}_{\mathrm{x}}\left[\mathrm{P}\left[W+S_{t}=j\right]\right] & \simeq \frac{1}{s}\left[\mathrm{P}\left[W^{(s, x)}+S_{t}^{(s, x)}=j\right]-\mathrm{P}\left[W+S_{t}=j\right]\right], \\
\text { and } \operatorname{IF}_{\mathrm{x}}[\varphi(u, t)] & \simeq \frac{1}{s}\left[\varphi^{(s, x)}(u, t)-\varphi(u, t)\right] .
\end{aligned}
$$

\subsection{Impact of database size on ERSM}

We proved the convergence to a centered Gaussian distribution of the rescaled difference between the "real" finite-time ruin probability starting from zero and its empirical equivalent, we obtained formulas to compute the asymptotic variance of this estimator (both for null and positive initial reserves), but one practical question immediately arises: how large should the size of the database be for the Normal approximation to be "good enough"? This is an important question to know from which range of database size the Normal approximation enables us to correctly approximate the Estimation-Risk Solvency Margin. To tackle this question, we plotted a few empirical distributions of the finite-time ruin probability for different values of database size $N_{D}$ and carried out several tests. Our finding is that the Normal approximation is of good quality for $N_{D} \geq 1000$ in our example, as the Gaussian hypothesis is not rejected for $N_{D} \geq 1000$ (see Tables 1 and 2 below).

In the example of Table 2, the finite-time ruin probability is 3.7\%, and the $95 \%$-reliable finite-time probability is around $4.8 \%$ for $N=1000$, which corresponds to a significant increase of $27 \%$.

In Table 3 we see that $\tilde{\Psi}_{1-\epsilon}^{O_{N} \text { reliable }}(u, t)$ is a quite good approximation of the empirical quantile of $\psi^{N}(u, t)$, as soon as $N$ is greater than 100, which is often true in practice. 


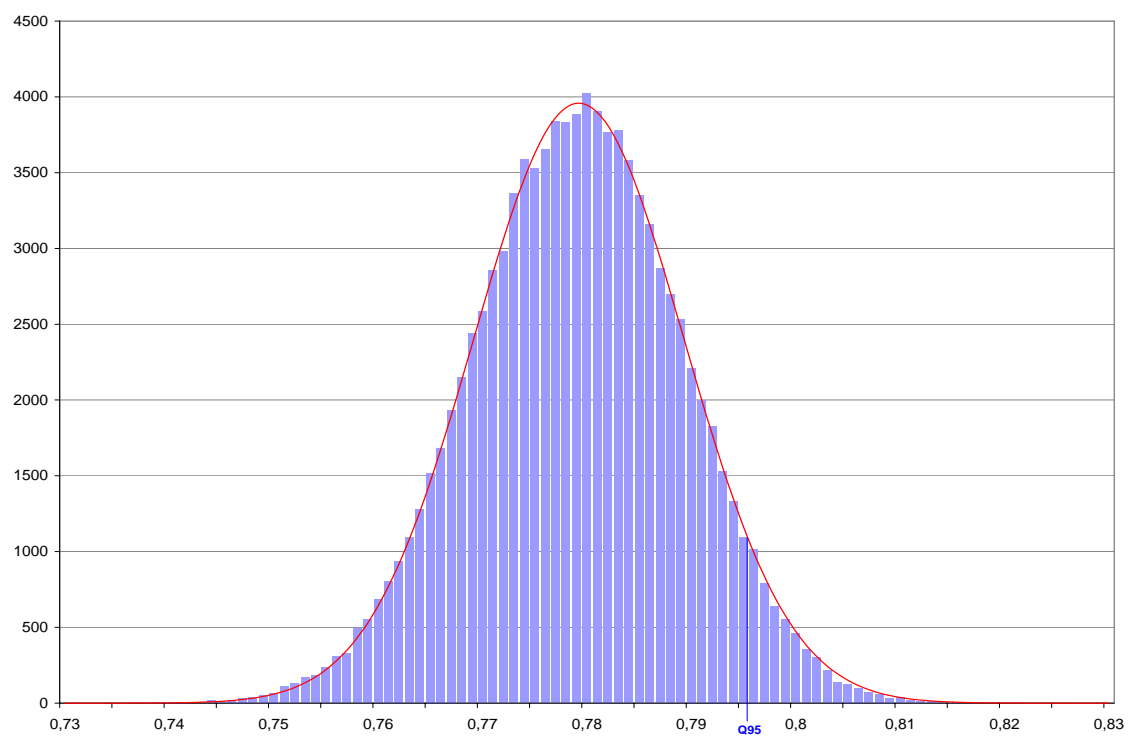

Figure 4. Histogram of 100000 empirical ruin probabilities $\psi^{N}(0, t)$ for $N=500$ and Gaussian p.d.f. with mean $\mu=\psi(u, t)$ and variance $V_{Y}\left[\lambda t \varphi^{(y)}(0, t)\right] / N, \lambda_{0}=1, c=1.1, t=10, \delta=1$.

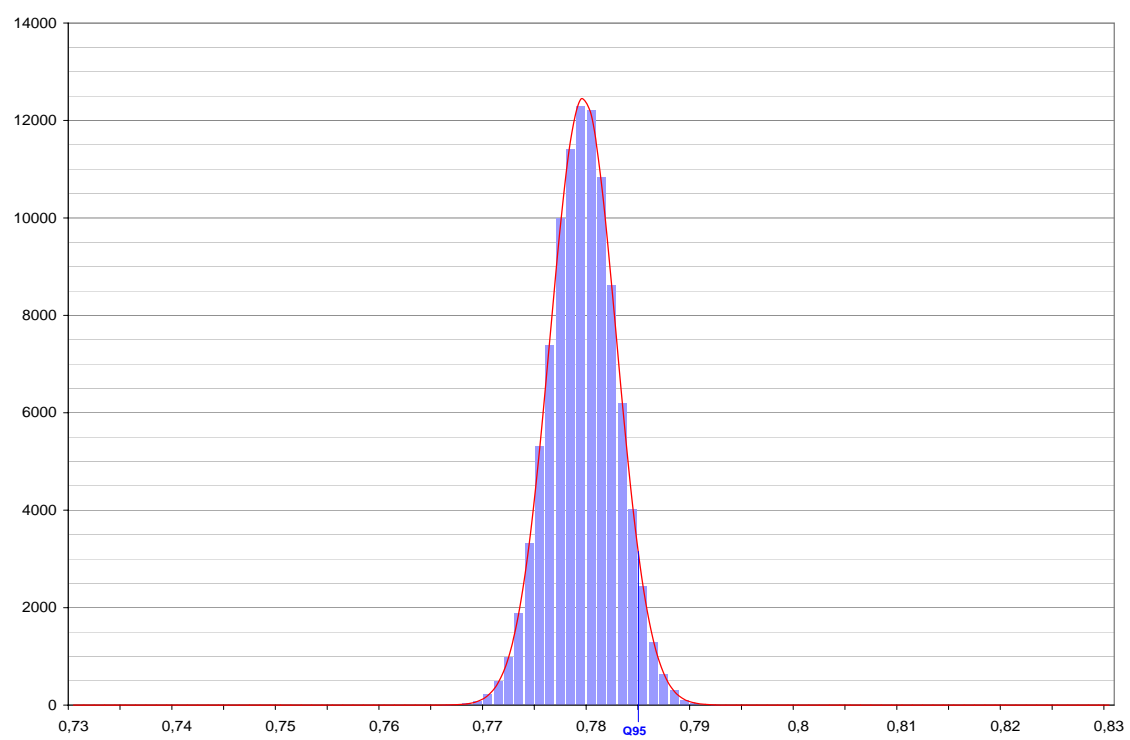

Figure 5. Histogram of 100000 empirical ruin probabilities $\psi^{N}(0, t)$ for $N=5000$ and Gaussian p.d.f. with mean $\mu=\psi(u, t)$ and variance $V_{Y}\left[\lambda t \varphi^{(y)}(0, t)\right] / N, \lambda_{0}=1, c=1.1, t=10, \delta=1$.

In Table 4 and Figure 8, we determine the smallest values of $u_{\eta}$ and $u_{\eta, \epsilon}$ such that $\psi\left(u_{\eta}, t\right)$ and $\left.\tilde{\psi}_{1-\epsilon \%}^{O_{N} \text { reliable }}\left(u_{\eta, \epsilon}, t\right)\right)$ are less than $\eta$. Due to the Normal approximation of $\psi^{N}(u, t)$ for $N \geq 1000$ (see Table 2), one can estimate here the $E R S M_{\eta, 1-\epsilon}$ by the difference $u_{\eta, \epsilon}-u_{\eta}$. We show that this margin is decreasing in the claim amount database size $N$.

In Table 5 we determine the values of $\eta$ that lead to values of $u_{\eta, 97.5 \%}$ of the same magnitude as $u_{0.5 \%}$. As $u_{0.5 \%}$ is the capital needed to control a classical $99.5 \%$ non-ruin probability, this gives us an idea of the confidence level $\eta$ that one should control to get results of the same magnitude as in the classical case, but with a stronger robustness, and more consistence with the impact of database size on estimation risk. 


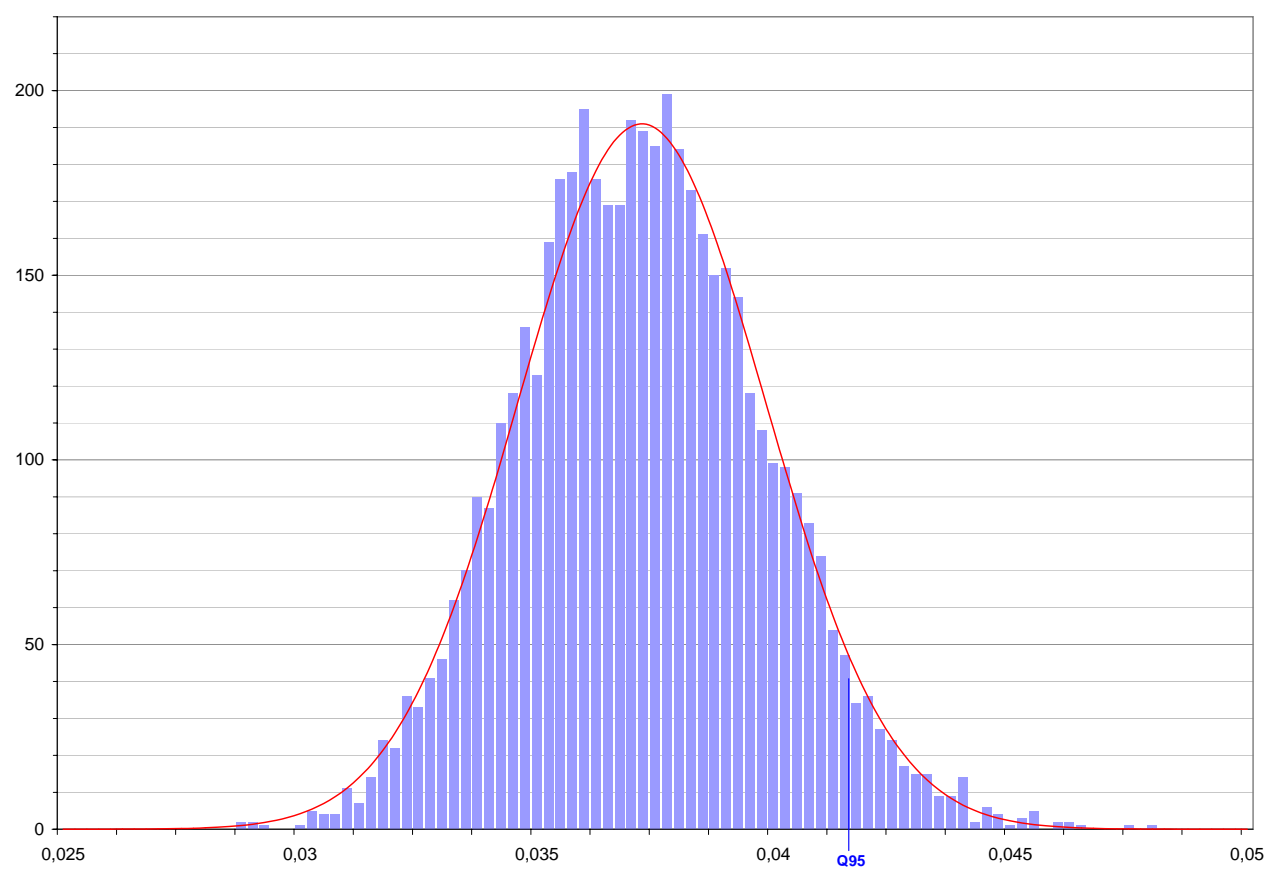

Figure 6. Histogram of 5000 empirical ruin probabilities $\psi^{N}(u, t)$ for $N=5000$ and Gaussian p.d.f. with mean $\mu=\psi(u, t)$ and variance $V_{Y}\left[\left[\operatorname{IF}_{Y}[\varphi(u, t)]\right] / N\right.$, $\lambda_{0}=1, c=1.1, t=10, u=10, \delta=1$.

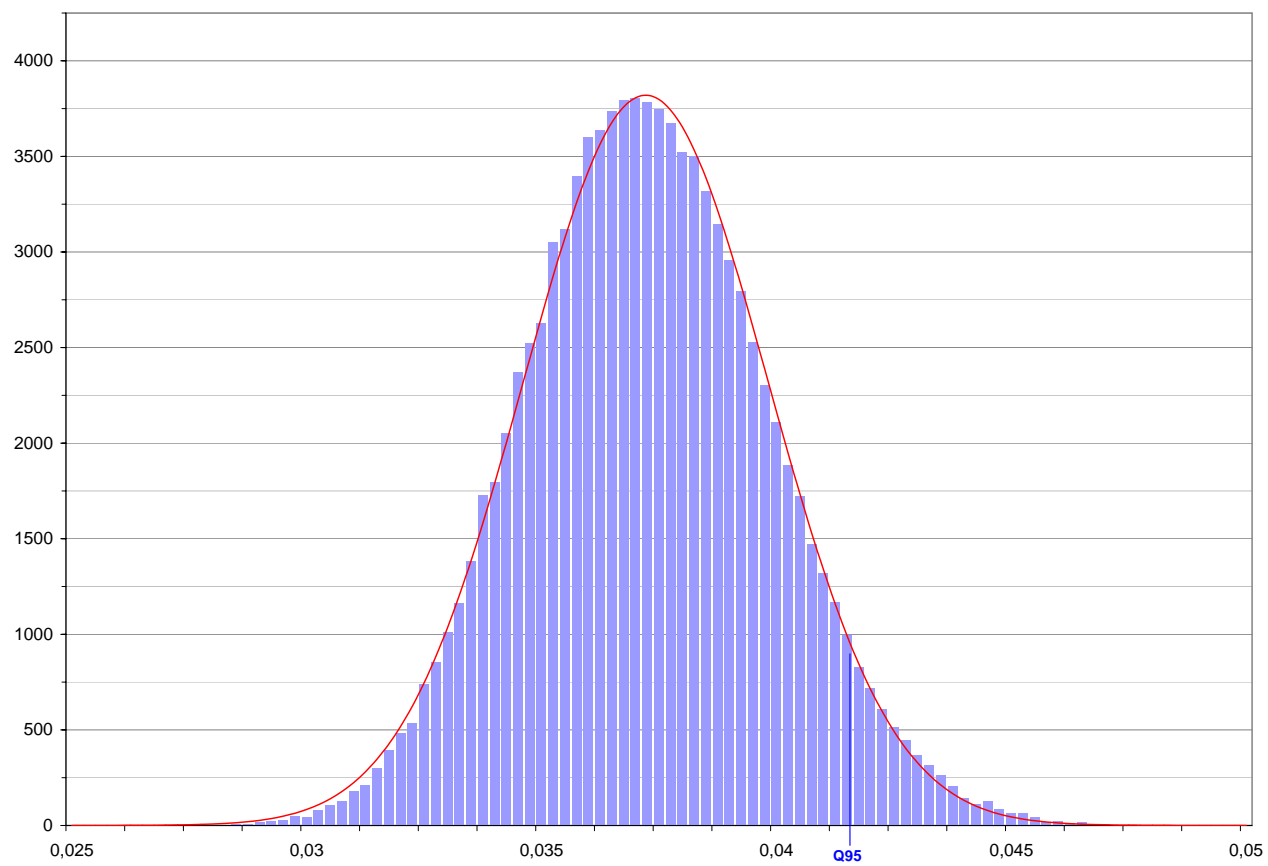

Figure 7. Histogram of 100000 empirical ruin probabilities $\psi^{N}(u, t)$ for $N=5000$ and Gaussian p.d.f. with mean $\mu=\psi(u, t)$ and variance $V_{Y}\left[\left[\operatorname{IF}_{Y}[\varphi(u, t)]\right] / N\right.$, $\lambda_{0}=1, c=1.1, t=10, u=10, \delta=1$. 


\begin{tabular}{|c|r|r|r|r|r|r|r|}
\hline$N$ & 1 & 10 & 100 & 1000 & 10000 & 100000 & 1000000 \\
\hline$\mu_{E}$ & 0.699139547 & 0.769697551 & 0.778242822 & 0.779687725 & 0.779726799 & 0.779714464 & 0.77972124 \\
$\sigma_{E}$ & 0.169347552 & 0.070979842 & 0.022666006 & 0.00714512 & 0.002253085 & 0.000708694 & 0.00022576 \\
$\mu$ & 0.779721532 & 0.779721532 & 0.779721532 & 0.779721532 & 0.779721532 & 0.779721532 & 0.779721532 \\
$\sigma$ & 0.225269457 & 0.071236457 & 0.022526946 & 0.007123646 & 0.002252695 & 0.000712365 & 0.000225269 \\
$\Delta_{\mu}$ & $-10.33471 \%$ & $-1.28558 \%$ & $-0.18965 \%$ & $-0.00434 \%$ & $0.00068 \%$ & $-0.00091 \%$ & $-0.00004 \%$ \\
$\Delta_{\sigma}$ & $-24.82445 \%$ & $-0.36023 \%$ & $0.61731 \%$ & $0.30144 \%$ & $0.01732 \%$ & $-0.51521 \%$ & $0.21763 \%$ \\
$D$ (KS stat.) & 0.45121 & 0.0856359 & 0.02129987 & 0.00853443 & 0.00831831 & 0.00831553 & 0.00837514 \\
p-value & $<0.001$ & $<0.001$ & 0.022 & $>0.250$ & $>0.250$ & $>0.250$ \\
\hline
\end{tabular}

Table 1

Empirical measures from 5000 values $\psi^{N}(0, t)$, and adequation to Gaussian distribution with parameters $\mu=\psi(0, t)$ and $\sigma^{2}=V_{Y}\left[\lambda t \varphi^{(Y)}(0, t)\right] / N . u=0, c=1.1, \delta=1, t=10$.

\begin{tabular}{|c|r|r|r|r|r|r|r|}
\hline$N$ & 1 & 10 & 100 & 1000 & 10000 & 100000 & 1000000 \\
\hline$\mu_{E}$ & 0.100446532 & 0.049993537 & 0.038233952 & 0.037476796 & 0.037375584 & 0.03732853 & 0.037344512 \\
$\sigma_{E}$ & 0.206272576 & 0.063201549 & 0.018060165 & 0.005807668 & 0.001849715 & 0.000589131 & 0.000183304 \\
$\mu$ & 0.037342766 & 0.037342766 & 0.037342766 & 0.037342766 & 0.037342766 & 0.037342766 & 0.037342766 \\
$\sigma$ & 0.184545163 & 0.058358305 & 0.018454516 & 0.00583583 & 0.001845452 & 0.000583583 & 0.000184545 \\
$\Delta_{\mu}$ & $168.98525 \%$ & $33.87743 \%$ & $2.38650 \%$ & $0.35892 \%$ & $0.08788 \%$ & $-0.03812 \%$ & $0.00468 \%$ \\
$\Delta_{\sigma}$ & $11.77349 \%$ & $8.29915 \%$ & $-2.13688 \%$ & $-0.48258 \%$ & $0.23104 \%$ & $0.95061 \%$ & $-0.67231 \%$ \\
$D$ (KS stat.) & 0.41984 & 0.26116 & 0.0561529 & 0.01292856 & 0.01014403 & 0.01558816 & 0.01022441 \\
p-value & $<0.001$ & $<0.001$ & $<0.001$ & $>0.250$ & $>0.250$ & 0.178 & $>0.250$ \\
$\psi_{5 \%}^{\text {reliable }}$ & 0.717433488 & 0.182651604 & 0.071621465 & 0.047605208 & 0.040501912 & 0.038294294 & 0.037645917 \\
\hline
\end{tabular}

Table 2

Empirical measures from 5000 values $\psi^{N}(u, t)$, and adequation to Gaussian distribution with parameters $\mu=\psi(u, t)$ and $\sigma^{2}=V_{Y}\left[\operatorname{IF}_{Y}[\varphi(u, t)]\right] / N . u=10, c=1.1, t=10, \delta=1$.

Table 3

\begin{tabular}{|c|r|r|r|}
\hline$N$ & empirical $\Psi_{1-\epsilon}^{O_{N} \text { reliable }}(u, t)$ & $\tilde{\Psi}_{1-\epsilon}^{O_{N} \text { reliable }}(u, t)$ & relative error \\
\hline 1 & 0,717433 & 0,340893 & $110,46 \%$ \\
10 & 0,182652 & 0,133334 & $36,99 \%$ \\
100 & 0,071621 & 0,067698 & $5,80 \%$ \\
1000 & 0,047605 & 0,046942 & $1,41 \%$ \\
10000 & 0,040502 & 0,040378 & $0,31 \%$ \\
100000 & 0,038294 & 0,038303 & $-0,02 \%$ \\
1000000 & 0,037646 & 0,037646 & $-0,001 \%$ \\
\hline
\end{tabular}

Comparison between the $95 \%$ empirical quantile of ruin probability $\Psi_{1-\epsilon}^{O_{N} \text { reliable }}(u, t)$ (from 5000 values $\left.\psi^{N}(u, t)\right)$ and the quantile $\tilde{\Psi}_{1-\epsilon}^{O_{N} r e l i a b l e}(u, t)$ of the Gaussian asymptotical distribution, $u=10, c=1.1, t=10, \delta=1, \epsilon=5 \%$.

\subsection{Convergence speed}

Here, we present results obtained by simulating ruin probabilities. Each ruin probability $\psi^{N}(u, t)$ is simulated as follows: first, we build one empirical distribution from $N$ claim amounts drawn from distribution $F$. Second, we compute the exact ruin probability as a functional of this empirical distribution. 
Table 4

\begin{tabular}{|c|r|r|r|}
\hline$N$ & $1-\epsilon=95 \%$ & $1-\epsilon=97.5 \%$ & $1-\epsilon=99.5 \%$ \\
\hline 1 & 22.17337 & 22.55869 & 23.22671 \\
100 & 17.26102 & 17.53763 & 18.07737 \\
500 & 16.21801 & 16.38931 & 16.73864 \\
1000 & 15.93426 & 16.07896 & 16.31359 \\
5000 & 15.42238 & 15.49750 & 15.64797 \\
10000 & 15.30943 & 15.36141 & 15.46433 \\
50000 & 15.16137 & 15.18416 & 15.22885 \\
100000 & 15.12662 & 15.14268 & 15.17413 \\
\hline$\infty$ & 15.04309 & 15.04309 & 15.04309 \\
\hline
\end{tabular}

Different values of $u_{\eta, \epsilon}$ such that $\tilde{\psi}_{1-\epsilon}^{O_{N}}$ reliable $\left(u_{\eta, \epsilon}, t\right)=\eta=0.5 \%, u=10, c=1.1, t=10, \delta=$ $1, \eta=0.5 \%$, and for $N=\infty$ value of $u_{\eta}$ such that $\psi\left(u_{\eta}, t\right)=\eta$.

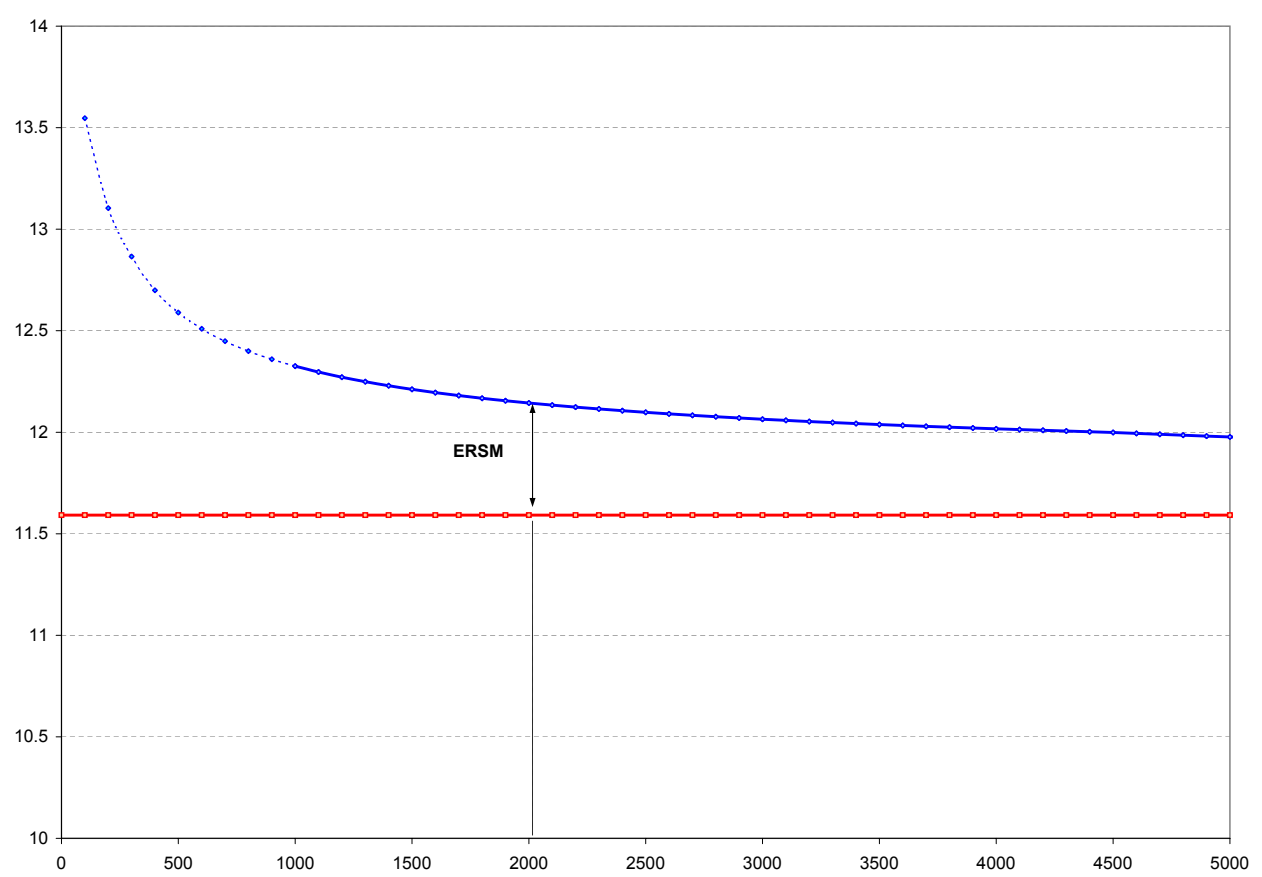

Figure 8. Values of $u_{\eta}$ and $u_{\eta, \epsilon}>u_{\eta}$ as functions of $N$, such that $\psi\left(u_{\eta}, t\right)=2 \%$ and $\tilde{\psi}_{1-\epsilon \%}^{O_{N} \text { reliable }}\left(u_{\eta, \epsilon}, t\right)=2 \%, \lambda_{0}=1, c=1.1, t=10, \delta=1, \epsilon=5 \%$.

Table 5

\begin{tabular}{|c|r|r|}
\hline$N$ & $\eta$ & ratio $\eta / 0.5 \%$ \\
\hline 1 & $8.59 \%$ & 17.19 \\
10 & $3.06 \%$ & 6.12 \\
100 & $1.31 \%$ & 2.62 \\
1000 & $0.76 \%$ & 1.51 \\
10000 & $0.58 \%$ & 1.16 \\
100000 & $0.53 \%$ & 1.05 \\
\hline
\end{tabular}

Values of $\eta$ such that $u_{\eta, 97.5 \%}=u_{0.5 \%} \cdot c=1.1, t=10, \delta=1$. 


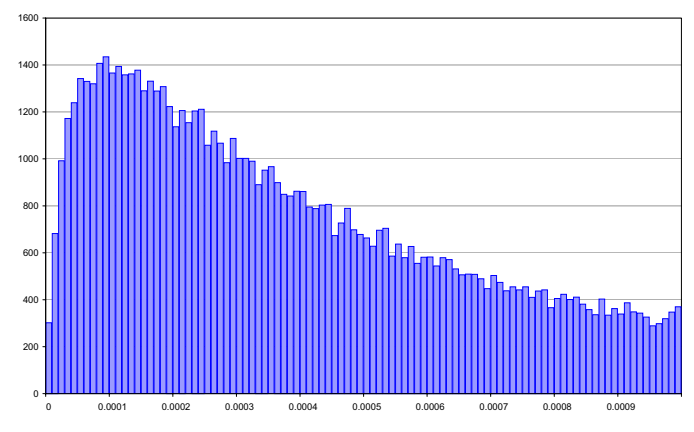

Figure 9. Histogram of 100000 empirical ruin probability $\psi^{N}(u, t)$ for $N=100$, $\lambda_{0}=1, c=1.1, t=10, u=20, \delta=1$.

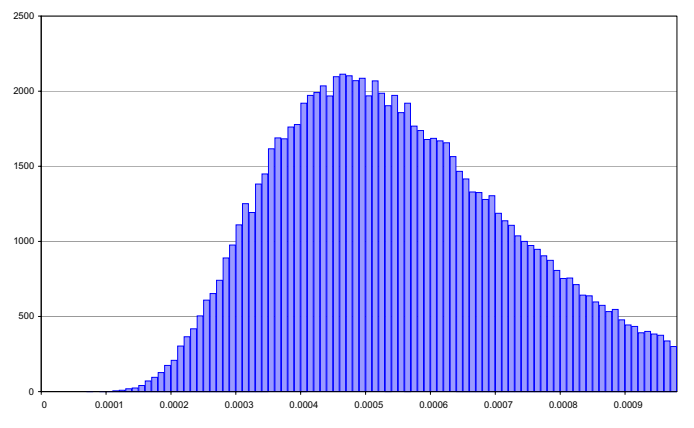

Figure 10. Histogram of 100000 empirical ruin probabilities $\psi^{N}(u, t)$ for $N=1000$, $\lambda_{0}=1, c=1.1, t=10, u=20, \delta=1$.

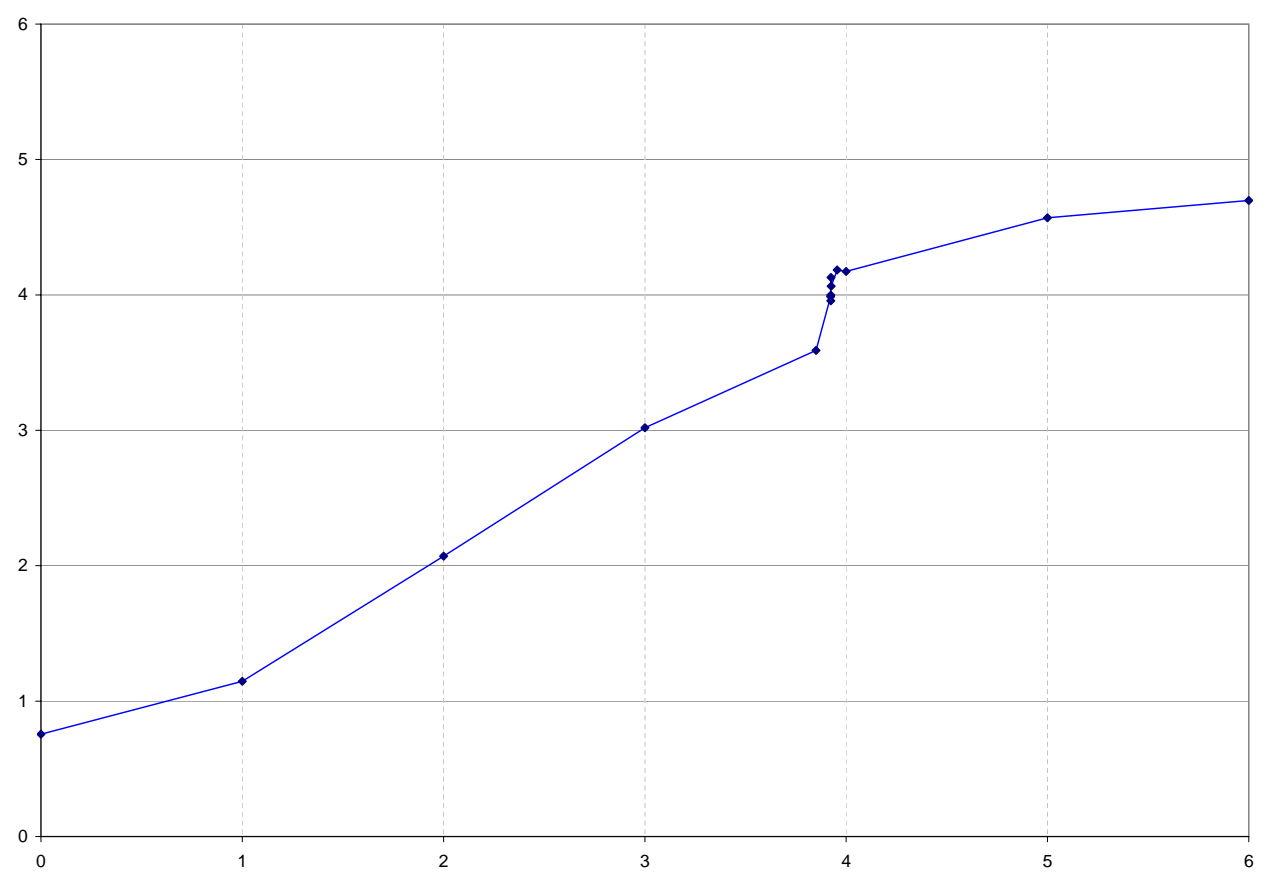

Figure 11. Values of distance $-\ln \left(D_{K S}\right)$ as a function of $\log _{10}(N)$, from 5000 values $\psi^{N}(u, t)$. $\lambda_{0}=1, c=1.1, t=10, u=15, \delta=1$.

For small ruin probabilities, we see in Figures 9 and 10 that the asymmetry of the empirical distributions of $\psi^{N}(u, t)$ would lead us to reject Normality for $N=100$ and 1000, and we can assume that the sample size needed to ensure the Gaussian hypothesis validation would be larger for smaller ruin probabilities. That is mainly what we try to quantify with further numerical analysis.

We have tried to quantify the empirical size $N$ from which, in our simulations, random variable $\psi^{N}(u, t)$ could be considered as a Gaussian random variable. In a first step, we have simulated 5000 values of $\psi^{N}(u, t)$, for various values of $N$. For each sample, we have computed the Kolmogorov-Smirnov distance $D_{K S}$ between the empirical distribution of the finite-ruin probability and its Gaussian asymptotical distribution. 


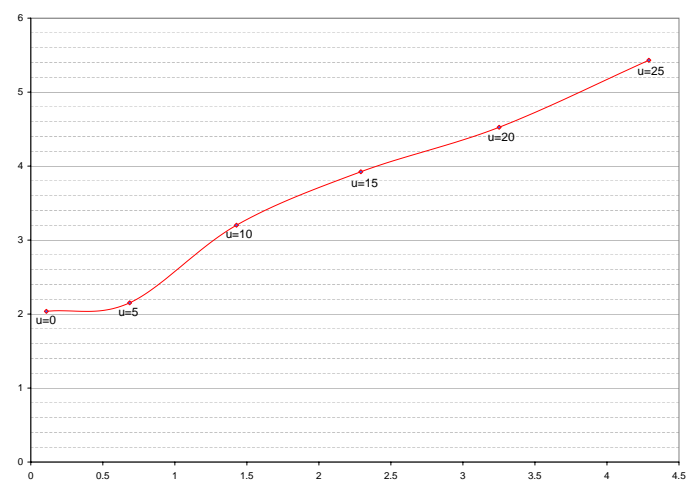

Figure 12. Values of $\log _{10}\left(N_{D}\right)$ as a function of $-\log _{10}(\psi(u, t))$. Exponential case. $\lambda_{0}=1, c=1.1, t=10, \delta=1$.

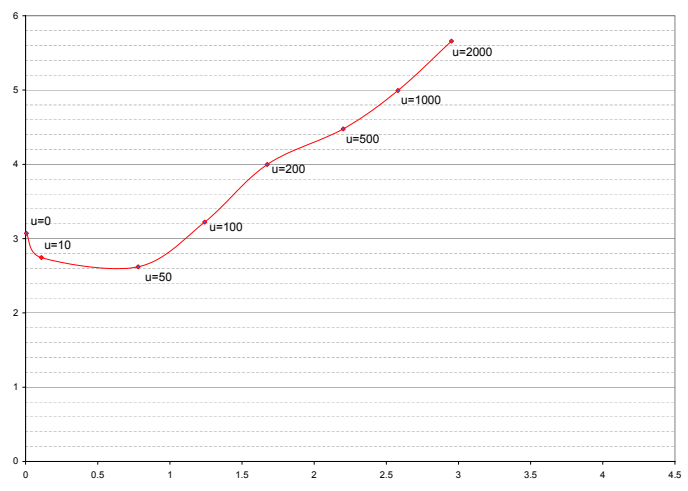

Figure 13. Values of $\log _{10}\left(N_{D}\right)$ as a function of $-\log _{10}(\psi(u, t))$. Pareto $(1,1.2)$ case. $\lambda_{0}=1, c=1.1, t=10, \delta=1$.

In Figure 11, we give the value of $-\ln \left(D_{K S}\right)$ as a function of $N$, for 5000 empirical $\psi^{N}(u, t)$; we see for example that $-\ln \left(D_{K S}\right)$ reaches the particular value 4 for every computed $N$ greater than a level $N_{D}$. From 5000 empirical ruin probabilities, validating Normality (with a $95 \%$ level significance level) leads to values of $-\ln \left(D_{K S}\right)$ approximately greater than 4 , and here to an empirical size $N_{D} \simeq 10^{3.924} \simeq 8400$. Of course, depending on simulations, this quantity may vary.

As a first approach, we have chosen to draw some values of $-\ln \left(D_{K S}\right)$ for a set of different values of initial reserves $u$. Since we did not observe situations where the barrier $-\ln \left(D_{K} S\right)=4$ was crossed more than once, we could determine one empirical $N_{D}$ by a dichotomic algorithm (with a total of around 14 computed points). We have then chosen to define $N_{D}$ as the first empirical value for which $-\ln \left(D_{K} S\right)$ was close enough to the target value, which gives an idea of the convergence rate. More rigorous formalization of this value $N_{D}$ would require the determination and the validation of a precise regression model, but a such model would require more simulations. We are just here trying to get rough indications on convergence speed.

In Figure 12 we have computed by this way the empirical size $N_{D}$ for which the Normality is validated for different values of initial reserves $u$. Since the ruin probability varies with $u$, we have given values of $\log _{10}\left(N_{D}\right)$ as a function of $-\log (\psi(u, t))$. As an example, for finite-time ruin probabilities of order $10^{-3}$, in this particular model (value 3 on horizontal axis), one may suppose that Normality is not validated for samples of size less than $10^{4.3} \simeq 20000$, whereas $10^{3.7} \simeq 5000$ might be enough for ruin probabilities of order $10^{-2}$. Note that data used for $u=1000$ is the same as the one in Table 2, but in this last application the distance $D_{K S}$ has been computed with unknown Gaussian parameters, causing the small difference with the one indicated in Table 2 . We finally insist on the fact that values of $N_{D}$ are just rough estimates and that this Figure only gives one empirical indication of the global need of larger samples to validate Normality for smaller ruin probabilities.

We also investigate the extreme case where claim amounts are Pareto-distributed. Pareto parameters are $a=1$ and $\alpha=1.2$ (with mean 6 and undefined standard deviation), and 99\%-percentile around 46.4. We always validate Normality in our simulations when $N$ is large enough. Nevertheless, we empirically see that, for a given ruin probability, this 
Table 6

\begin{tabular}{|c|r|r|r|r|}
\hline$\delta$ & $V_{u}$ & time PC1 & time PC2 & $\begin{array}{r}\text { PC2 + interpolation } \\
\text { (relative error on Vu) }\end{array}$ \\
\hline 1 & 0.03405692 & $0.031 \mathrm{~s}$ & $0.016 \mathrm{~s}$ & - \\
0.5 & 0.04251800 & $0.047 \mathrm{~s}$ & $0.016 \mathrm{~s}$ & - \\
0.25 & 0.04879227 & $0.11 \mathrm{~s}$ & $0.06 \mathrm{~s}$ & - \\
0.125 & 0.05271634 & $0.72 \mathrm{~s}$ & $0.4 \mathrm{~s}$ & - \\
0.1 & 0.05357748 & $1.5 \mathrm{~s}$ & $0.97 \mathrm{~s}$ & - \\
0.05 & 0.05538387 & $18.7 \mathrm{~s}$ & $12.1 \mathrm{~s}$ & - \\
0.025 & 0.05633120 & $4 \mathrm{~min} 29 \mathrm{~s}$ & $2 \mathrm{~min} 55 \mathrm{~s}$ & $9.3 \mathrm{~s}(0.22 \%)$ \\
0.0125 & 0.05681639 & $70 \mathrm{~min}$ & $44 \mathrm{~min} 33 \mathrm{~s}$ & $1 \mathrm{~min} 10 \mathrm{~s}(0.22 \%)$ \\
\hline
\end{tabular}

Computation times to get $V_{u}$ and the whole set of $\operatorname{IF}_{\mathrm{x}}\left[\varphi\left(u, \frac{n}{c}\right)\right], x \in \delta N . u=10, c=1.1$, $t=10$.

validation requires larger values of $N$ than in the exponential case.

From this numerical analysis, it appears that for small ruin probabilities, particularly for heavy tailed claim amounts, large values of $N$ are required to validate Normality. If Normality was assumed without any data, the asymmetry of bootstrapped ruin probability and the reliable ruin probability would probably be underestimated.

\subsection{Computation times}

The program which computes influence functions of ruin probabilities and bootstrapped ruin probabilities has been written in $\mathrm{C}++$, with a standard 64-bit double-precision arithmetic. One central procedure has been optimized with only 13 assembly code instructions, in order to avoid unnecessary access to principal memory (but without using further optimization processes like loop unrolling, cache prefetching or SIMD instructions). Computations were carried out on an older single AMD Athlon processor (year 2001), 1330MHz, with 512Mo PC2100 RAM (label PC1), and on a more recent single Intel Pentium 4 processor (year 2002), 2660MHz, with 1024Mo PC2700 RAM (label PC2).

We give here in Table 6 the time required to compute $\operatorname{IF}_{\mathrm{x}}\left[\varphi\left(u, \frac{n}{c}\right)\right]$, for $x$ varying from 0 to $u+n$ by step $\delta$, for a varying discretization step $\delta$ ( $\delta$ is the monetary unit, and thus impacts $u$ and $n$ too). This time is also the one needed to compute the exact value of $V_{u}$ with Proposition 8 or 20 and with Formula (21). Here, since $u$ and $n$ are of the same order, computation times are similar (but using both methods provides a useful validation of numerical results).

Since $\delta$ impacts $x, u$ and $n$, the complexity of the calculation of one $\operatorname{IF}_{\mathrm{x}}\left[\varphi\left(u, \frac{n}{c}\right)\right]$ is roughly proportional to $1 / \delta^{3}$, so that the global complexity for all $x \in \delta \mathbb{N}, x<u+n$, is roughly proportional to $1 / \delta^{4}$. This may involve computational difficulties for small values of $\delta$.

Nevertheless, we show in the last column of Table 6 that a simple linear interpolation of $\operatorname{IF}_{\mathrm{x}}\left[\varphi\left(u, \frac{n}{c}\right)\right]$ for any $x \in\left[k x_{0},(k+1) x_{0}\right], k \in \mathbb{N}$, leads to reasonable errors and faster computation times (we have chosen in this Table $x_{0}=20 \delta$ for $u=0.025$ and $x_{0}=40 \delta$ 
for $u=0.0125)$.

\section{Conclusion}

We have provided algorithms to compute influence functions of finite-time ruin probabilities. We have also proved the weak convergence of a sequence of empirical finite-time ruin probabilities starting from zero initial reserve toward a Gaussian random variable, and numerical investigation seems to confirm that the result holds for $u>0$, which is important for applications. We hope to be able to prove this in the near future. We defined the concepts of reliable finite-time ruin probability and Estimation Risk Solvency Margin (ERSM). Results on influence functions ensure us that the proposed estimators of these quantities are robust (i.e. their influence functions are bounded). Numerical results show that our method is implementable, even if some numerical problems may occur in the case of very heavy tails or very small discretization step and would deserve more attention.

\section{References}

Bening, V. and Korolev, V. (2000), Nonparametric estimation of the ruin probability for generalized risk processes, Theory of probability and its applications, Vol. 47-1, 1-16.

Billingsley, P. (1999), Convergence of probability measures, Wiley Series in Probability and Statistics: Probability and Statistics, Second Edition.

Croux, K. and Veraverbeke, N. (1990), Nonparametric estimators for the probability of ruin, Insurance: Mathematics and Economics, Vol. 9-2,3, 127-130.

De Vylder, F. E. (1997), La formule de Picard-Lefèvre pour la probabilité de ruine en temps fini, Bulletin Français d'Actuariat, Vol. 1,2, 31-40.

De Vylder, F. E.(1999), Numerical Finite-Time Ruin Probabilities by the Picard-Lefèvre Formula, Scandinavian Actuarial Journal, Vol. 2, 97-105.

Frees, E. (1986), Nonparametric estimation of the probability of ruin, Astin Bulletin, Vol. $16,81-90$.

Gerber H.-U. (1979), Introduction to mathematical risk theory, Huebner Foundation Monograph.

Gotze, F. (1984), Expansions for Von Mises functionals, Zeitschrift für Warscheinlichkeitstheorie und verwandte Gebiete, 65, 599-625.

Hampel, F. (1974), The influence curve and its role in robust estimation, Journal of the American Statistical Association, Vol. 69, 383-393.

Hampel, F.R., Ronchetti, E.M., Rousseeuw, P.J., Stahel, W.A. (1986), Robust Statistics. The Approach Based on Influence Functions, Wiley Series in Probability and Statistics, Wiley, New York.

Hipp, C. (1989), Estimators and bootstrap confidence intervals for ruin probabilities, Astin Bulletin, Vol. 19-1, 57-70.

Hoeffding, W. (1948), A class of statistics with asymptotically normal distribution, The annals of Mathematical Statistics, Vol. 19, No. 3, 293-325.

Huber, P.J. (1981), Robust Statistics, Wiley Series in Probability and Statistics, Wiley, New York. 
Ignatov G., Kaishev V.K., Krachunov R.S. (2001), An improved finite-time ruin probabilities formula and its Mathematica implementation, Insurance: Mathematics and Economics 29, 375-386.

Marceau, E. and Rioux, J. (2001), On robustness in risk theory, Insurance: Mathematics and Economics 29, 167-185.

Mazza C. and Rullière (2004), A link between wave governed random motions and ruin processes, Insurance: Mathematics and Economics 35, 205-222.

Panjer H. (1981), Recursive evaluation of a family of compound distributions, Astin Bulletin, Vol. 12, 22-26.

Panjer H. , Wang S. (1993), On the stability of recursive formulas, Astin Bulletin Vol. 23,2 .

Picard P., Lefèvre C. (1997), The Probability of Ruin in Finite Time with Discrete Claim Size Distribution, Scandinavian Actuarial Journal, Vol. 1, 58-69.

Picard P., Lefèvre C. (1998), The moments of ruin time in the classical risk model with discrete claim size distribution, Insurance: Mathematics and Economics 23, 157-172.

Picard P., Lefèvre C. and Coulibaly I. (2003a), Problèmes de ruine en théorie du risque à temps discret avec horizon fini, J. Appl. Probab. 40, no. 3, 527-542.

Picard P., Lefèvre C. and Coulibaly I. (2003b), Multirisks model and finite-time ruin probabilities, Methodol. Comput. Appl. Probab. 5, no. 3, 337-353.

Rullière D. and Loisel S. (2004), Another look at the Picard-Lefèvre formula for finite-time ruin probabilities, Insurance: Mathematics and Economics 35, 187-203.

Seal H. L. (1969), Stochastic Theory of a Risky Business, New York: Wiley.

Takács L. (1962a), A generalization of the ballot problem and its application in the theory of queues, J. Amer. Statist. Assoc. 57, 327-337.

Takács L. (1962b), The time dependence of a single-server queue with Poisson input and general service times, Ann. Math. Statist. 33, 1340-1348.

Von Mises, R. (1947), On the asymptotic distribution of differentiable statistical functions, The annals of mathematical statistics, Vol. 18-3, 309-348. 


\section{A Appendix}

\section{A.1 Sensitivity analysis}

In the continuous case, one may get the following formulas for partial derivatives of $\varphi(0, t)$.

Proposition 17 For $u, c, t>0$ such that $f_{W}$ is continuously differentiable on $[0, u+c t]$,

$$
\begin{aligned}
\frac{\partial}{\partial c} \varphi(0, t) & =\int_{0}^{t}\left(\frac{t-x}{t}\right) \frac{\partial}{\partial c} f_{S_{t / c}(x)} d x \\
\frac{\partial}{\partial \lambda} \varphi(0, t) & =\int_{0}^{t}\left(\frac{t-x}{t}\right) \frac{\partial}{\partial \lambda} f_{S_{t / c}(x)} d x .
\end{aligned}
$$

This may be extended to $k^{\text {th }}$ order partial derivatives for $k \geq 2$.

In the case of discrete claim size distribution, set $h_{j}(\tau)=\mathrm{P}\left[S_{\tau}=j\right]$ for $\tau \geq 0$ and $j \in \mathbb{N}$, and $\Pi_{i}=\mathrm{P}[W=i]$ for $i \in \mathbb{N}$, with $\pi_{0}=0$. Panjer's formula gives that

$$
h_{j}(t)=\sum_{i=1}^{j} \lambda t \frac{i}{j} \Pi_{i} h_{j-i}(t),
$$

so that, for $k \geq 1$,

$$
\frac{\partial^{k}}{\partial \lambda^{k}} h_{j}(\tau)=\sum_{i=1}^{j} \frac{i \Pi_{i}}{j} t\left(\lambda \frac{\partial^{k}}{\partial \lambda^{k}} h_{j-i}(t)+k \frac{\partial^{k-1}}{\partial \lambda^{k-1}} h_{j-i}(t)\right),
$$

where

$$
\frac{\partial^{k}}{\partial \lambda^{k}} h_{0}(t)=(-t)^{k} e^{-\lambda t}
$$

This last relation permits the computation of the derivatives of $h_{j}(\tau)$ with respect to $\lambda$ recursively. In the particular case where $k=1$, one obtains

$$
\frac{\partial}{\partial \lambda} h_{j}(t)=\frac{1}{\lambda} h_{j}(t)+\sum_{i=1}^{j} \frac{i \Pi_{i}}{j} t\left(\lambda \frac{\partial}{\partial \lambda} h_{j-i}(t)\right) .
$$

Proposition 18 For all $t>0$ and for any Borelian $A \subset \mathbb{R}$, the following identities hold:

$$
\begin{gathered}
\frac{\partial}{\partial \lambda} \mathrm{P}\left[S_{t} \in A\right]=t \mathrm{P}\left[S_{t}+W \in A\right]-t \mathrm{P}\left[S_{t} \in A\right] ., \quad k \geq 1, \\
\frac{\partial^{k}}{\partial \lambda^{k}} \mathrm{P}\left[S_{t} \in A\right]=t^{k} \sum_{i=0}^{k} C_{k}^{i}(-1)^{k-i} \mathrm{P}\left[S_{t}+W^{* i} \in A\right], \quad k \geq 0,
\end{gathered}
$$

where $W^{* i}$ corresponds to a sum of $i$ independent copies of $W$.

Proof: Check that for given $t>0$ and $n \in \mathbb{N}$,

$$
\frac{\partial}{\partial \lambda} \mathrm{P}\left[N_{t}=n\right]=t \mathrm{P}\left[N_{t}=n-1\right]-t \mathrm{P}\left[N_{t}=n\right], \text { with } \mathrm{P}\left[N_{t}<0\right]=0
$$


But $\mathrm{P}\left[S_{t} \in A\right]=\sum_{n \in \mathbb{N}} \mathrm{P}\left[N_{t}=n\right] \mathrm{P}\left[W^{* n} \in A\right]$, and the result follows. Extension to higher derivative orders holds since

$$
\frac{\partial^{k}}{\partial \lambda^{k}} \mathrm{P}\left[N_{t}=n\right]=t^{k} \sum_{i=0}^{k} C_{k}^{i}(-1)^{k-i} \mathrm{P}\left[N_{t}=n-i\right],
$$

with $\mathrm{P}\left[N_{t}=-1\right]=0$.

\section{A.2 Influence functions}

We give here alternative formulas from Picard and Lefèvre (1997) for the non-ruin probability:

$$
\varphi\left(u, \frac{n}{c}\right)=\sum_{i=0}^{u} h_{u-i}(-i) \cdot \widetilde{H}_{i+n}(i+n)
$$

or

$$
\varphi\left(u, \frac{n}{c}\right)=H_{u}(n)+\sum_{i=0}^{u} h_{u-i}(-i)\left(\tilde{H}_{i+n}(i+n)-\tilde{H}_{i}(i+n)\right) .
$$

As a direct consequence, we get the following alternative recursive schemes for the influence function of the finite-time non-ruin probabilities $\varphi(u, t), u \in \mathbb{N}$.

Recall that $\operatorname{IFH}_{\mathrm{x}, j}(\tau)=\operatorname{IF}_{\mathrm{x}}\left[\tilde{H}_{j}(\tau)\right]$. We have

$$
\operatorname{IFH}_{\mathrm{x}, j}(\tau)=\sum_{i=0}^{j} \operatorname{IF}_{\mathrm{x}, i}(\tau)\left(1-\frac{i}{\tau}\right)
$$

with $\operatorname{IFH}_{\mathrm{x}, 0}(0)=0$. In particular, $\operatorname{IFH}_{\mathrm{x}, n}(n)=\operatorname{IF}_{\mathrm{x}}\left[\varphi\left(0, \frac{n}{c}\right)\right], n \in \mathbb{N}$.

\section{Proposition 19 (IF for $\varphi(u, t)$ - first method)}

$$
\begin{aligned}
\operatorname{IF}_{\mathrm{x}}\left[\varphi\left(u, \frac{n}{c}\right)\right] & =\sum_{i=0}^{u+n} \operatorname{IF}_{\mathrm{x}, i}(n)-\sum_{k=1}^{n} \operatorname{IF}_{\mathrm{x}, u+k}(k) \tilde{H}_{n-k}(n-k) \\
& -\sum_{k=1}^{n} h_{u+k}(k) \operatorname{IFH}_{\mathrm{x}, n-k}(n-k)
\end{aligned}
$$

Proof: Taking the derivative of (8) for the contaminated single amount distribution, setting $s=0$ and applying then (6) gives the required result.

\section{Proposition 20 (IF for $\varphi(u, t)$ - second method)}

$$
\begin{aligned}
\operatorname{IF}_{\mathrm{x}}\left[\varphi\left(u, \frac{n}{c}\right)\right]= & \sum_{i=0}^{u} \operatorname{IF}_{\mathrm{x}, u-i}(-i) \sum_{j=0}^{i+n} h_{j}(i+n)\left(1-\frac{j}{i+n}\right) \\
& +\sum_{i=0}^{u} h_{u-i}(-i) \sum_{j=0}^{i+n} \operatorname{IF}_{\mathrm{x}, j}(i+n)\left(1-\frac{j}{i+n}\right) .
\end{aligned}
$$


Proof: As above, take the derivative of (A.4) for the contaminated single amount distribution, set $s=0$ and use (6).

\section{Proposition 21 (IF for $\varphi(u, t)$ - third method)}

$$
\begin{aligned}
\operatorname{IF}_{\mathrm{x}}\left[\varphi\left(u, \frac{n}{c}\right)\right]= & \sum_{i=0}^{u} \operatorname{IF}_{\mathrm{x}, i}(n)+\operatorname{IF}_{\mathrm{x}, u-i}(-i) \sum_{k=i+1}^{i+n} h_{k}(i+n)\left(1-\frac{k}{i+n}\right) \\
& +\sum_{i=0}^{u} h_{u-i}(-i) \sum_{k=i+1}^{i+n} \mathrm{IF}_{\mathrm{x}, k}(i+n)\left(1-\frac{k}{i+n}\right) .
\end{aligned}
$$

Proof: Again, take the derivative of (A.5) for the contaminated single amount distribution, set $s=0$ and use (6).

\section{Proposition 22 (IF for $\varphi(u, t)$ - direct recursion)}

$$
\begin{aligned}
\operatorname{IF}_{\mathrm{x}}\left[\varphi\left(u, \frac{n}{c}\right)\right]= & -\frac{\lambda n}{c} \mathrm{P}\left[S_{n / c}+W \leq u+n\right]+\frac{\lambda n}{c} \varphi\left(u, \frac{n}{c}\right) \\
& +\sum_{k=1}^{n} \frac{\lambda k}{c} \mathrm{P}\left[S_{k / c}+W=u+k\right] \varphi\left(0, \frac{n-k}{c}\right) \\
& -\sum_{k=1}^{n} \mathrm{P}\left[S_{k / c}=u+k\right] \operatorname{IF}_{\mathrm{x}}\left[\varphi\left(0, \frac{n-k}{c}\right)\right] .
\end{aligned}
$$

Proof: Use the partial derivative of $\varphi\left(u, \frac{n}{c}\right)$ with respect to $\lambda$, as given in (3), and simplify derivatives of the distribution of aggregate claim amounts using (A.3). Finally, use the expression of $\varphi\left(u, \frac{n}{c}\right)$ as given in (8) to simplify some terms, and the result follows.

In order to find the initial reserve $u \in \mathbb{R}^{+}$respecting some constraints for ruin probabilities and influence functions, the following adaptation of previous formulas may be useful. Write $\epsilon_{u}=u-[u], \epsilon_{n}=n-[n], \nu=\left[\epsilon_{u}+\epsilon_{n}\right]$, where the brackets denote the integer part. We have for $u>0, n>0$,

$$
\begin{aligned}
\operatorname{IF}_{\mathrm{x}}[\varphi(u, n / c)] & =\sum_{i=0}^{[u+n]} \operatorname{IF}_{\mathrm{x}, i}(n)-\sum_{k=1}^{[n]+\nu} \operatorname{IF}_{\mathrm{x},[u]+k}\left(k-\epsilon_{u}\right) \tilde{H}_{[n]-k+\nu}\left(n-k+\epsilon_{u}\right) \\
& -\sum_{k=1}^{[n]+\nu} h_{[u]+k}\left(k-\epsilon_{u}\right) \operatorname{IFH}_{\mathrm{x},[n]-k+\nu}\left(n-k+\epsilon_{u}\right),
\end{aligned}
$$

and the following adaptation of proposition 20,

$$
\begin{aligned}
\operatorname{IF}_{\mathrm{x}}[\varphi(u, n / c)]= & \sum_{i=0}^{[u]} \operatorname{IF}_{\mathrm{x},[u]-i}\left(-i-\epsilon_{u}\right) \tilde{H}_{[n]+i+\nu}\left(n+i+\epsilon_{u}\right) \\
& +\sum_{i=0}^{[u]} h_{[u]-i}\left(-i-\epsilon_{u}\right) \operatorname{IFH}_{\mathrm{x},[n]+i+\nu}\left(n+i+\epsilon_{u}\right) .
\end{aligned}
$$

